\title{
Application of the Hopfield Neural Network in Routing for Computer Networks
}

\author{
by \\ Adel Abdullah Smeda \\ A Thesis Submitted to the \\ Faculty of Engineering \\ in Partial Fulfillment of the Requirements for the Degree of

\section{MASTER OF APPLIED SCIENCE}

Major Subject: Electrical and Computer Engineering

Dalhousie University, DalTech

Halifax, Nova Scotia 
National Library

of Canada

Acquisitions and

Bibliographic Services

395 Wellington Street

Ottawa ON K1A ON4

Canada
Bibliothèque nationale du Canada

\section{Acquisitions et}

services bibliographiques

395, rue Wellington

Ottawa ON KIA ON4

Canada

Your tile Votre relerence

Our file Notre rèlerence

The author has granted a nonexclusive licence allowing the National Library of Canada to reproduce, loan, distribute or sell copies of this thesis in microform, paper or electronic formats.

The author retains ownership of the copyright in this thesis. Neither the thesis nor substantial extracts from it may be printed or otherwise reproduced without the author's permission.
L'auteur a accordé une licence non exclusive permettant à la Bibliothèque nationale du Canada de reproduire, prêter, distribuer ou vendre des copies de cette thèse sous la forme de microfiche/film, de reproduction sur papier ou sur format électronique.

L'auteur conserve la propriété du droit d'auteur qui protège cette thèse. $\mathrm{Ni}$ la thèse ni des extraits substantiels de celle-ci ne doivent être imprimés ou autrement reproduits sans son autorisation. 


\title{
“AUTHORITY TO DISTRIBUTE MANUSCRIPT THESIS"
}

\begin{abstract}
TITLE:
Application of Hopfield Neural Network in Routing for Computer Networks
\end{abstract}

The above library may make available or authorize another library to make available individual photo/microfilm copies of this thesis without restrictions.

Full Name of Author:

Adel A. Smeda

Signature of Author:

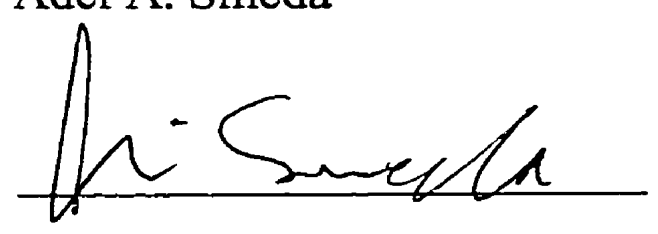

Date:

April 1,1999 


\section{Table of Contents}

List of Figures.........................................................v

List of Tables.................................................................vi

List of Abbreviations and Symbols........................................viii

Acknowledgments............................................................ix

Abstract....................................................................x

Chapter 1 Introduction....................................................1

1.1 Objective and Scope of the thesis................................................

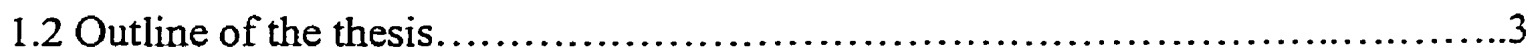

Chapter 2 Routing in Data Networks......................................5

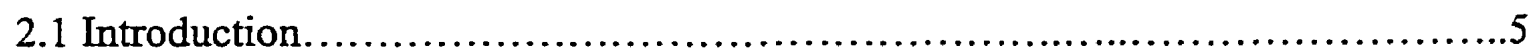

2.2 Network Algorithms and Shortest Path Routing.............................

2.2.1 Undirected Graphs....................................................

2.2.2 Minimum Weight Spanning Trees ....................................9

2.2.3 Shortest Path Problem...................................................

2.3 Shortest Path Algorithms................................................. 14

2.3.1 The Bellman-Ford Algorithm ..........................................14

2.3.2 Dijkstra's Algorithm.................................................. 17

2.3.3 The Floyd-Warshall Algorithm.......................................21

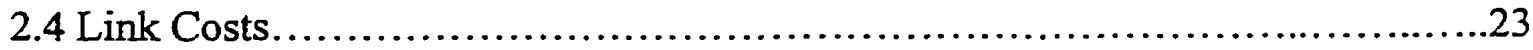


Chapter 3 Neural Networks.

3.1 What are Neural Networks, and What are They good for?....................27

3.2 Neural Network for Optimization......................................28

Chapter 4 Neural Network and Routing.................................34

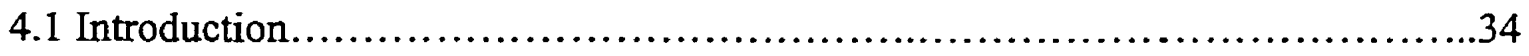

4.2 Routing Using The Hopfield Neural Network..............................35

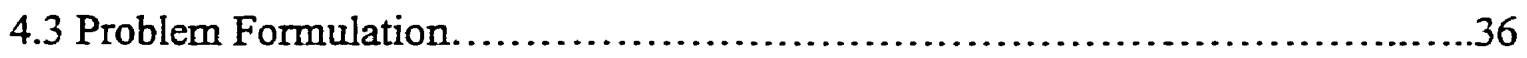

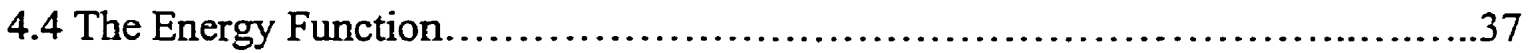

4.5 The Weight Matrix and The Biases...................................... 41

4.6 Simulation Results of the Hopfield Module................................44

4.6.1 Choosing the Values of $T_{1}, T_{2}, T_{3}, T_{4}$, and Neural Transfer Parameter $\lambda . .44$

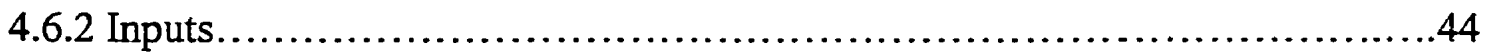

4.6.3 Activations, Outputs, and Their Updating...............................45

4.7 Comparison Between Neural Network Based Algorithm and Conventional

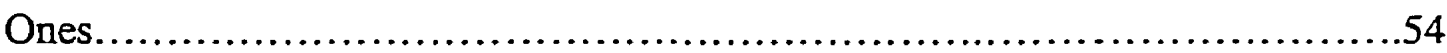

Chapter 5 Conclusions and Future Research.........................57

5.1 Suggestions for Future Research........................................ 58

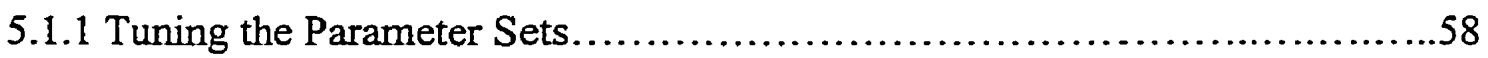

5.1.2 Alternative Neural Network Structures .................................58

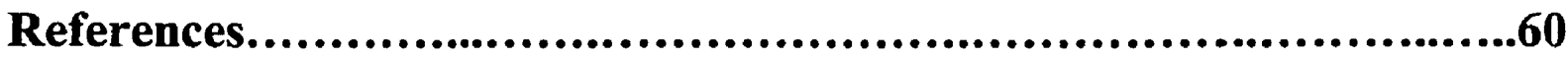

Appendix A: Complete Output of a five-node network and a six-node network.............................................................63 


\section{List of Figures}

Figure 2.1 Routing in a datagram network........................................6

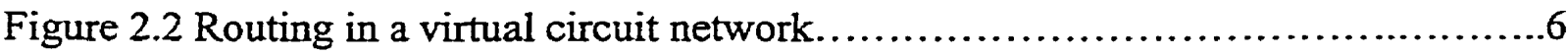

Figure 2.3 Example of graphs......................................................

Figure $2.4 \mathrm{~A}$ graph and three subgraphs.............................................

Figure 2.5 Minimum weight spanning tree construction..............................12

Figure 2.6 Counter example for distributed MST construction with nondistinct arc

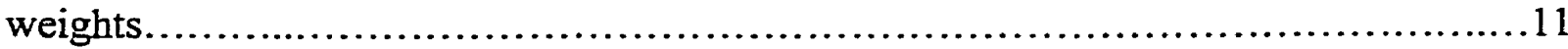

Figure 2.7 Representation of a directed graph..................................... 14

Figure 2.8 Successive iterations of the Bellman-Ford method...........................17

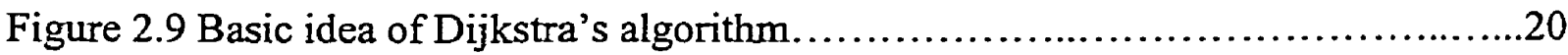

Figure 2.10 Example using the Bellman-Ford and Dijkstra's algorithms................21

Figure 3.1 A generic processing element...............................................

Figure 3.2 Hopfield Neural Network ................................................ 30

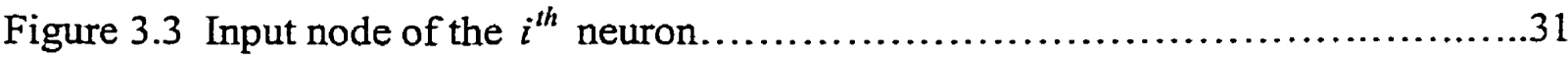

Figure 4.1 An illustrative example of the routing problem and the neural output...........41

Figure 4.2 A five-node network with link costs...................................47

Figure 4.3 A six-node network...................................................48

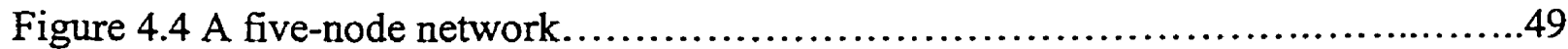

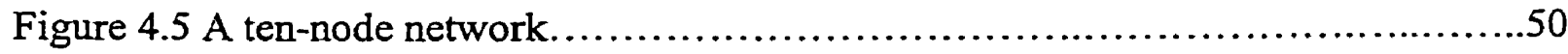

Figure 4.6 Network with 14 nodes ................................................52

Figure 5.1 Optimal route percentage for different networks...........................58

Figure 5.2 Self organising map from $\mathrm{T}$. Kohonen....................................59 


\section{List of Tables}

Table 4.1 Neural network solution for five-node network...........................47

Table 4.2 The neural module output for six-node network.........................48

Table 4.3 Routing table for five-node network when source $=1$ and destination $=5 \ldots \ldots . .49$

Table 4.4 Performance of the proposed module vs. Cavalier's........................51

Table 4.5 Performance of the proposed module vs. Cavalier's.......................51

Table 4.6 Summary of simulation results for fourteen-node network.................52

Table 4.7 Comparison of all non optimal routes with optimal routes for fourteen-node

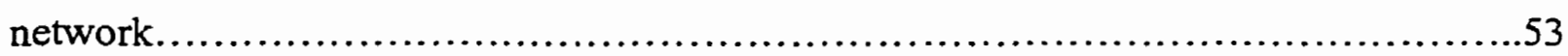

Table 4.8 Comparison of the neural approach and other routing algorithms..............56 


\section{List of Abbreviations and Symbols}

\begin{tabular}{|c|c|}
\hline$A N N$ & Artificial Neural Network \\
\hline $\mathrm{HNN}$ & Hopfield Neural Network \\
\hline TSP & Traveling Salesman Problem \\
\hline ETANN & Electronic Trainable Artificial Neural Networks \\
\hline$\phi$ & Empty set \\
\hline MST & Minimum Weight Spanning Tree \\
\hline$D_{i}^{h}$ & Distance (cost) from node $i$ to node $h$ \\
\hline$D_{i}$ & Distance from each node $i$ to node 1 \\
\hline SP & Shortest Path \\
\hline$W_{i j}$ & Connectivity between neurons $i$ and $j$ \\
\hline$U_{i}$ & Neural network input of the ith neuron \\
\hline$V_{i}$ & Neural network output of the ith neuron \\
\hline$I_{i}$ & External Input to a node $i$ \\
\hline$\lambda$ & Gain parameter \\
\hline$P^{s d}$ & Path connecting $s$ to $d$ \\
\hline$L^{s d}$ & Length of a path connecting $s$ to $d$ \\
\hline$C_{i j}$ & Cost of sending data on link $(i, j)$ \\
\hline$P_{i j}$ & Probability that link $(i, j)$ is usable \\
\hline$\tau$ & Time constant \\
\hline$\delta$ & Kronecker delta function \\
\hline
\end{tabular}


$\Delta t$

$\Delta u_{i j}$

Time step

Change in the activation 


\section{Acknowledgements}

It is my pleasure to acknowledge the help that I received while working on this thesis. I would like to express my gratitude to Dr. El-Hawary for his guidance, supervision, and patience over the past two years.

Thanks to the Libyan Ministry of Education for their financial support. Also, thanks to my parents, many relatives, and friends who helped me in one way or another along way.

Finally, thanks to my loving wife, I can not thank her enough for her constant support, understanding, and encouragement. Truly she shared my success and frustration. This dissertation is dedicated to her. 


\section{Abstract}

Given a network in which data packets are being routed along communication links between nodes making up the network, the problem arises of how to determine the optimal paths to route the packet through traffic. In determining the optimal path, links are assumed to have a cost representing the desirability of using that particular link to route a data packet. The optimal path then becomes the path in which the total link cost from a source node to a destination node is minimized. While there are many conventional solutions available, none of them are without flaws [1]. In order to find a better solution, the possibility of using an artificial neural network of some type as a routing algorithm is explored in this thesis. The neural network chosen to solve the routing problem is the Hopfield Neural Network. This approach is based on a solution proposed for the Traveling Salesman Problem (TSP) as given in [2]. The routing problem is considerably similar to the TSP problem in that it is concerned with finding an optimal route which travels between a source node and a destination node. The neural network architecture is implemented and tested. A comparison between the neural network solution and conventional routing algorithms is also presented. The neural network gives approximately $97.3 \%$ of optimal routes, this represents a significant improvement over other neural network approaches already implemented. 


\section{Chapter 1}

\section{Introduction}

From a topological viewpoint, computer networks can be seen as multipath systems in the sense that there is often no single path between a source node, to which the computer generating the information is connected, and a destination node. In packet switched networks, the main need is to optimize the paths connecting the source and the destination, which can be achieved by using a suitable routing algorithm. This optimization takes account of various parameters such as the length of the paths, the traffic in the various links, and the length of the queues, which are summarized in a cost parameter associated to each communication link.

There are many routing algorithms in use with different levels of sophistication and efficiency [3]. Routing can be done in a centralized, distributed, or localized manner. In centralized algorithms, all route choices are made at a central node, while in distributed algorithms, the computation of routes is shared among the network nodes with information exchanged between nodes as necessary. In localized routing algorithms, each node needs to have the current network connectivity and computes the routes to all possible destination nodes based on this connectivity information. In order to have the most current network connectivity, all network nodes broadcast their connectivity to neighboring nodes to each other. The Dijkstra's algorithm [3] can be considered as a localized algorithm. 
The centralized routing method requires a special node in the network which periodically receives information from all other network nodes and, based on this global information, it sets up and updates routing tables for all nodes. This method suffers from high communication overhead and reliability problems, since failure of the central control node results in shutdown of the entire network.

The distributed routing approach can reduce some problems of the centralized routing. In this case, each node makes its own routing decisions based on the local information it receives from its neighboring nodes. Looping of packets and deadlocks might happen due to inconsistent routing paths. The most commonly used distributed routing algorithm is the distributed Bellman-Ford algorithm [3].

In the localized routing algorithm, such as the Dijkstra's algorithm, all network nodes broadcast their network connectivity to neighbor nodes, so that each network node can react quickly to changes in the network, but does increase the communication cost of broadcasting such changes.

Either centralized, distributed, or localized routing algorithms can be operated in a static or adaptive manner. In static routing algorithms, the path used by each origin-destination pair is fixed regardless of traffic conditions and network changes. In adaptive routing algorithms, the paths used to route a new packet between source and destination change occasionally in response to traffic conditions and network changes, i.e., failed links, increase or decrease of link cost.

The recent resurgence of interest in neural networks has its roots in the recognition that the brain performs computation in a different manner than conventional digital computers. Computers are extremely fast and precise at executing sequences of instructions designed for specific algorithms. A human information processing system is 
composed of many neurons (i.e., processing elements) switching at speeds about a million times slower than computer gates. Yet humans are more efficient than computers at computationally complex tasks such as speech understanding, visual recognition, etc. Artificial neural networks are designed to exploit this unique computational power of the human brain.

From the technology point of view, Very Large Scale Integration (VLSI) circuits can put tens-to-hundreds of processing elements in one chip, such as Intel Electronic Trainable Artificial Neural Networks (ETANN) 80170NX [4]. For some problems, the neural VLSI chip can indeed produce solutions better and faster than the conventional approach. It is the intent of this thesis to investigate the possibility of using a neural network to solve the routing problem. For a communication network, each node can be equipped with a neural network, and all network nodes can train and later use the neural networks to obtain optimal or near-optimal routes at the same time. In other words, all neural networks work collectively to solve the routing problem.

\subsection{Objective and Scope of the Thesis}

The objective of this thesis is to study the use of neural networks to solve the problem of routing in packet switched computer networks. In this thesis, the routing problem has been approached considering the Hopfield Neural Network [2] used as an optimizer. The Hopfield neural network would seem to be suitable for solving optimization problems like the routing problem, although it is remarkably critical in the phase devoted to mapping the conditions surrounding the problem to be optimized with the internal parameters of the network itself. This approach is particularly powerful if we refer to a hardware implementation which guarantees low implementation cost and short processing time. 


\subsection{Outline of the Thesis}

The following Chapter describes the routing problem and some of the conventional algorithms used, including Bellman-Ford algorithm, Dijkstra's algorithm, and FloydWarshall algorithm, and the remainder of the chapter is dedicated to the link cost issue. Chapter 3 introduces the concept of Artificial Neural Networks. The Hopfield Neural Network is also explained in this Chapter. In Chapter 4 Hopfield model for routing is given in detail, the output from testing the model with different networks is presented. At the end of Chapter 4 a comparison between the Hopfield neural network model and some conventional algorithms is given. Finally Chapter 5 includes concluding remarks and suggestions for further research. The complete output of a five-node network and a sixnode network are given in Appendix-A. 


\section{Chapter 2}

\section{Routing in Data Networks}

\subsection{Introduction}

The routing algorithm is frequently referred to as the network layer protocol that guides packets through the communication subnet to their correct destination. The times at which routing decisions are made depend on whether the network uses datagrames or virtual circuits. In a datagram network two successive packets of the same user pair may travel along different routes, and a routing decision is necessary for each individual packet (Figure 2.1). In a virtual circuit network, a routing decision is made when each virtual circuit is set up. The routing algorithm is used to choose the communication path for the virtual circuit. All packets of the virtual circuit subsequently use this path up to the time the virtual circuit is either terminated or rerouted for some reason (Figure 2.2).

Routing in a network typically involves a rather complex collection of algorithms that work more or less independently and yet support each other by exchanging services or information. The complexity is due to a number of reasons. First, routing coordination between all the nodes of the subnet rather than just a pair of modules as, for example, in data link and transport layer protocols. Second, the routing system must cope with link and node failures, requiring redirection of traffic and an update of the databases maintained by the system. Third, to achieve high performance, the routing algorithm may need to modify its routes when some areas within the network become congested [3]. 


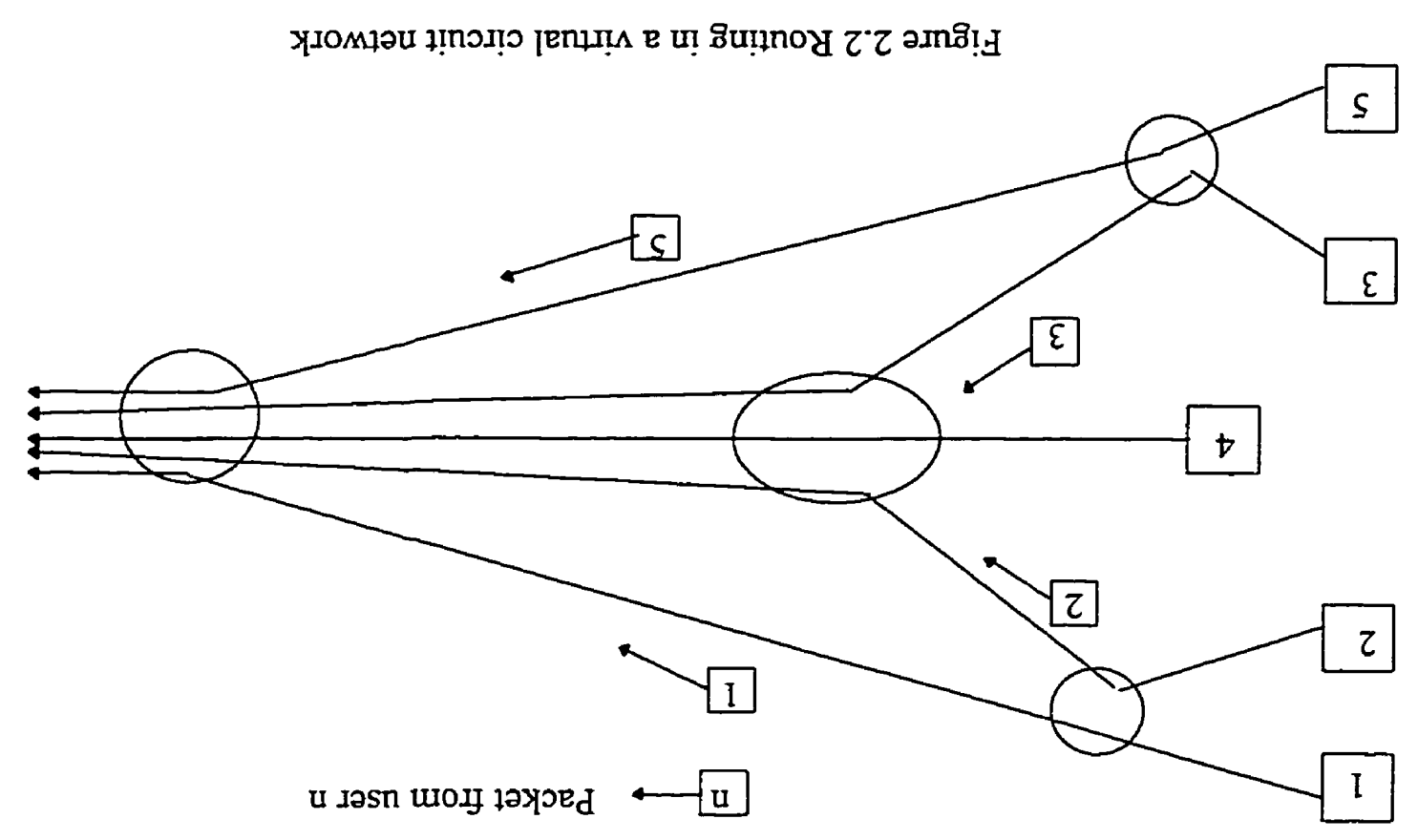

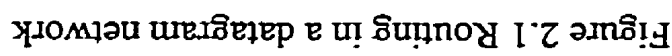

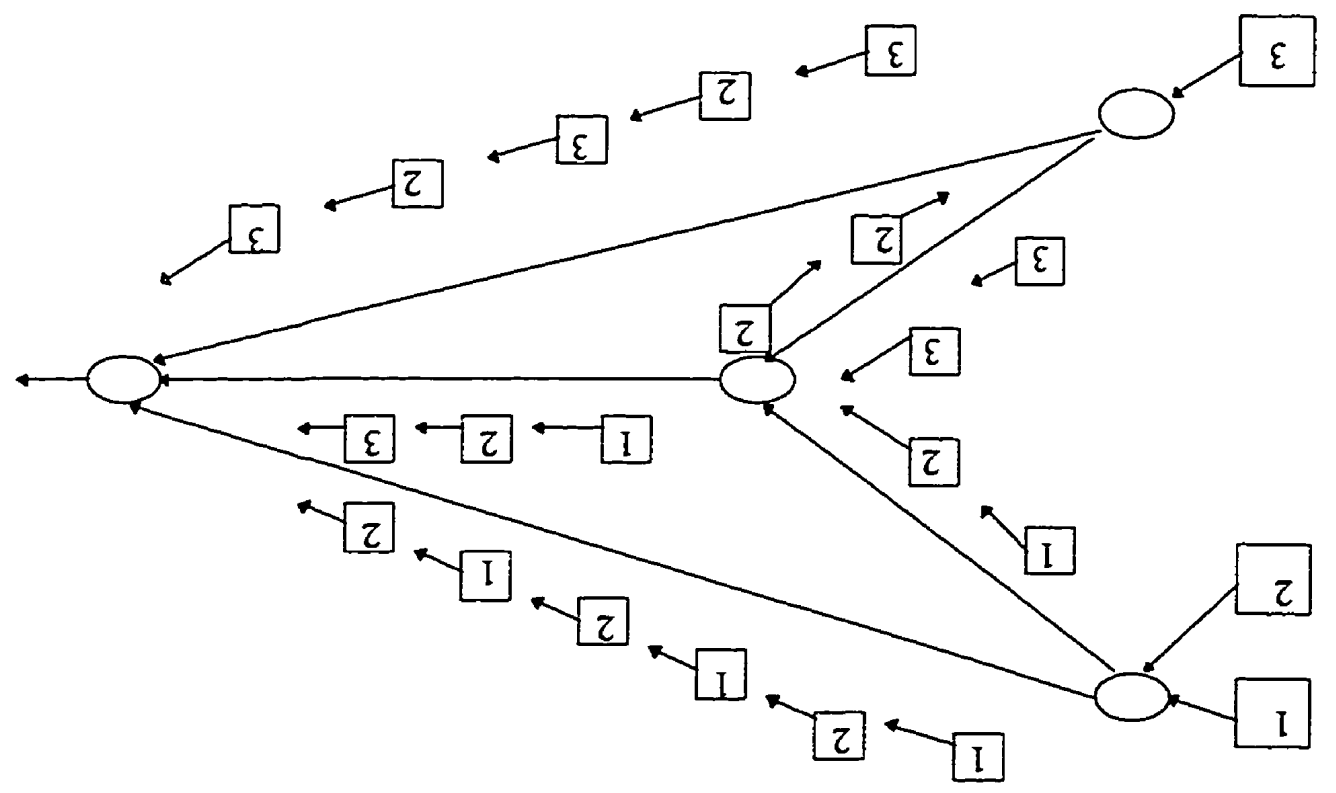

u Iasn woy дәу⿰冫е d 


\subsection{Network Algorithms and Shortest Path Routing}

Routing methods involve the use of a number of simple graph theoretic problems such as the shortest path problem. In this section we consider shortest path routing in detail together with related problems of interest. We start by introducing some basic graph theoretic notions.

\subsubsection{Undirected Graphs}

A graph, $G=(N, A)$, is defined to be a finite nonempty set $N$ of nodes and a collection $A$ of pairs of distinct nodes from $N$. Each pair of nodes in $A$ is called an arc or a link. Several examples of graphs are given in Figure 2.3. The major purpose of the formal definition $G=(N, A)$ is to stress that the location of the nodes and the shapes of the arcs in a pictorial representation are totally irrelevant. If $n_{1}$ and $n_{2}$ are nodes in a graph and $\left(n_{1}, n_{2}\right)$ is an arc, this arc is said to be incident on $n_{1}$ and $n_{2}$ [3].

A walk in a graph $G$ is a sequence of nodes $\left(n_{1}, n_{2}, \ldots, n_{e}\right)$ such that each of the pairs $\left(n_{1}, n_{2}\right),\left(n_{2}, n_{3}\right), \ldots,\left(n_{e-1}, n_{e}\right)$ are arcs of $G$. A walk with no repeated node is a path. A walk $\left(n_{1}, n_{2}, \ldots, n_{e}\right)$ with $n_{1}=n_{e}, e>3$, and no repeated nodes other than $n_{1}=n_{e}$ is called a cycle [3]. These definitions are illustrated in Figure 2.4.

We say that a graph is connected if for each $i$ there is a path $\left(i=n_{1}, n_{2}, \ldots, n_{e}=j\right)$ to each other node j. Note that the graphs in Figure 2.3 (a) and (c) are connected, whereas the graph in Figure 2.3 (b) is not connected. We spot an absence of connectivity in a graph by seeing two sets of nodes with no arcs between them. 


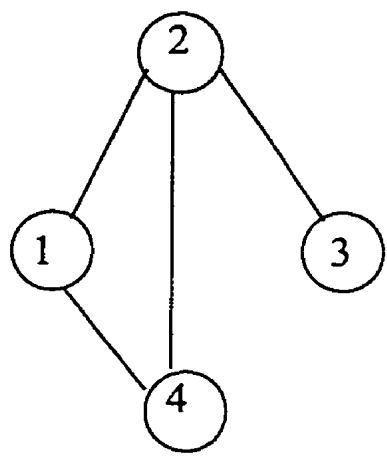

(a)
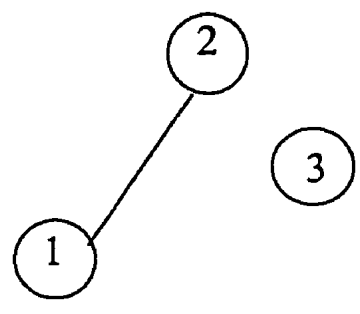

1

3
$\mathrm{N}=\{1,2,3,4\}$

$\mathrm{A}=\{(1,2),(2,3),(4,1),(4,2)\}$ (b)

$N=\{1,2,3\}$
$A=\{(1,2)\}$ (c) $\mathrm{N}=\{1\}$ $\mathrm{A}=\{\}$

Figure 2.3 Example of graphs with a set of nodes $\mathrm{N}$ and a set of $\operatorname{arcs} \mathrm{A}$

A tree is a connected graph that contains no cycles. A spanning tree of a graph $G$ is a subgraph of $G$ that is a tree and that includes all the graph in Figure 2.4 (a).

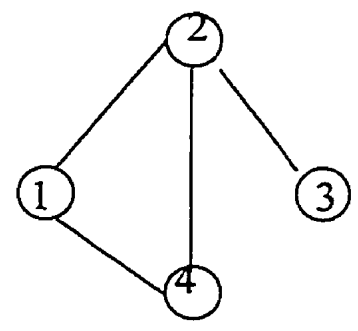

(a)

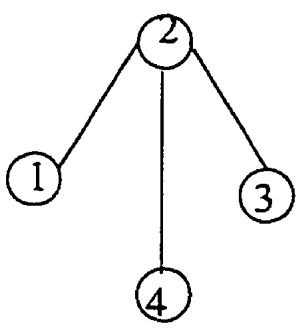

(b)

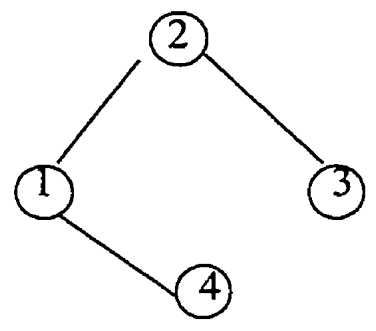

(c)
(2)

(3)

(d)

Figure 2.4 A graph (a) and three subgraphs (b), (c), and (d) 


\subsubsection{Minimum Weight Spanning Trees}

It is possible to construct a spanning tree rooted at some node by sending a packet from the node to all its neighbors; the neighbors must send the packet to their neighbors, and so on, with all nodes marking the transmitter of the first packet they receive as their "father" on the tree. However, such a tree has no special properties. If the communication cost of different links should be taken into account in constructing a spanning tree, we may consider using a minimum weight spanning tree, which we now define.

Consider a connected graph $G=(N, A)$ with $N$ nodes and $A$ arcs, and a weight $W_{i, j}$ for each arc $(i, j) \in A$. A minimum weight spanning tree (MST for short) is a spanning tree that represents the communication cost of a message along the arc in either directions, and the total spanning tree weight represents the cost of broadcasting a message to all nodes along the spanning tree.

Any subtree (i.e., a subgraph that is a tree) of an MST will be called a fragment. Note that a node by itself is considered a fragment. An arc having one node in a fragment and the other node not in this fragment is called an outgoing arc from the fragment. The following proposition is of central importance for MST algorithm [3].

Proposition 1: Given a fragment $F$, let $\alpha=(i, j)$ be a minimum weight outgoing arc from $F$, where the node $j$ is not in $F$. Then $F$, extended by arc $\alpha$ and node $j$ is a fragment.

Proposition 1 can be used as the basis for MST construction algorithms. The idea is to start with one or more disjoint fragments and enlarge or combine them by adding minimum weight outgoing arcs. One method (the prim-Dijkstra algorithm) starts with an arbitrarily selected single node as a fragment and enlarges the fragment by successively adding a minimum weight outgoing arc. Another method (Kruskal's algorithm) starts 
with each node being a single node fragment; it then successively combines two of the fragments by using the arc that has minimum weight over all arcs that when added to the current set of fragments do not form a cycle ( see Figure 2.5). Both of these algorithms terminate in $N-1$ iterations.

Kruskal's algorithm [3] proceeds by building up simultaneously several fragments that eventually join into an MST; however, only one arc at a time is added to the current set of fragments. Proposition 1 suggests the possibility of adding a minimum weight outgoing arc simultaneously to each fragment in a distributed algorithm manner. This is possible if there is a unique MST.

A distributed algorithm for constructing the MST in a graph with a unique MST is as follows [3]. Start with a set of fragments (these can be the nodes by themselves, for example). Each fragment determines its minimum weight outgoing arc, adds it to itself, and forms the fragment that lies at the other end of this arc. It can be seen that as long as the arc along which two fragments join is indeed a minimum weight outgoing arc for some fragment, the algorithm maintains a set of fragments of the MST at all times, and no cycle is ever formed. Furthermore, new arcs will be added until there are no further outgoing arcs and there is only one fragment (by necessity the MST). Therefore, the algorithm cannot stop short of finding the MST. Indeed, for the algorithm to work correctly, it is not necessary that the procedure for arc addition be synchronized for all fragments. What is needed, however, is a scheme for the nodes and arcs of a fragment to determine the minimum weight outgoing arc. There are a number of possibilities along these lines, but it is of interest to construct schemes that accomplish this with low communication overhead. A distributed MST algorithm of the type just described has been used in the PARIS network [5]. 


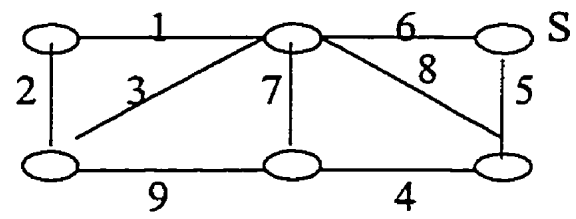

(a)<smiles>[CH-]C=C</smiles>

(1)

(2)

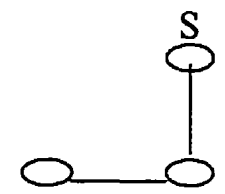

(3)

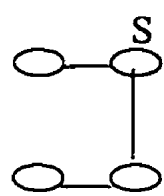

(4)

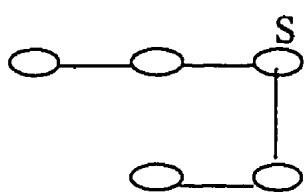

(5)

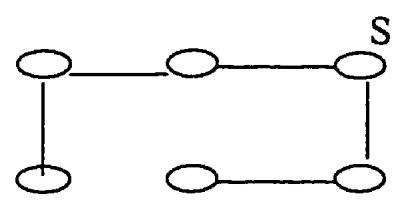

(6)

(b)

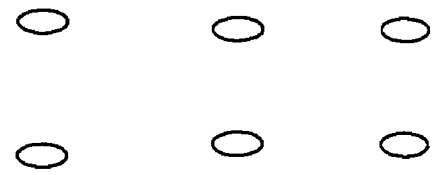

(1)

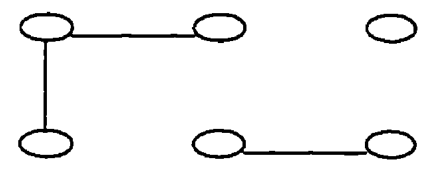

(4)

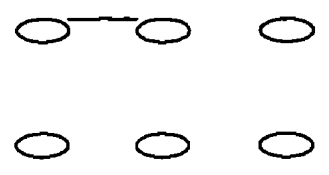

(2)

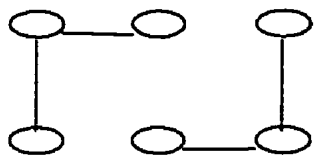

(5)

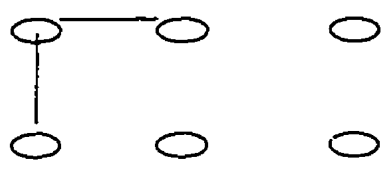

(3)

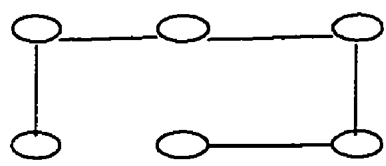

(6)

(c)

Figure 2.5 Minimum weight spanning tree construction. (a) Graph with arc weights as indicated. (b) Successive iterations of the Prim-Dijkstra algorithm. The starting fragment consists of the single node marked $\mathrm{S}$. The fragment is successively extended by adding a minimum weight outgoing arc. (c) Successive iterations of the Kruskal algorithm. The algorithm starts with all nodes as fragment. At each iteration, we add the arc that has minimum weight out of all arcs that are outgoing from one of the fragments. 
To see what can go wrong in the case of nonunique MST, consider the triangular network of Figure 2.6 where all arc lengths are unity. If we start with the three nodes as fragments and allow each fragment to add to itself an arbitrary, minimum weight outgoing arc, there is the possibility that the arcs $(1,2),(2,3)$, and $(3,1)$ will be added simultaneously by nodes 1,2 , and 3 , respectively, with a cycle resulting.

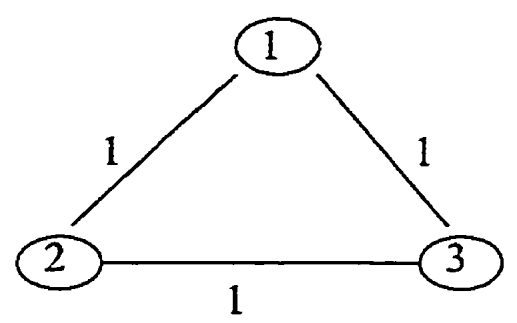

Figure 2.6 Counter example for distributed MST construction with nondistinct arc weights. Nodes 1,2 , and 3 can add simultaneously to their fragments arcs $(1,2),(2,3)$, and $(3,1)$, respectively, with a cycle resulting.

Consider now an MST problem where the arc weights are not distinct. The ties between arcs with the same weight can be broken by using the identities of their nodes; that is, if arcs $(i, j)$ and $(k, l)$ with $i<j$ and $k<l$ have equal weight, prefer $\operatorname{arc}(i, j)$ if $i<k$ or if $i=k$ and $j<l$, and prefer arc $(k, l)$ otherwise. Thus, by implementing, if necessary, the scheme just described, one can assume without loss of generality that the weights are distinct and that the MST is unique, thereby allowing the use of distributed MST algorithms that join fragments along minimum weight outgoing arcs.

\subsubsection{Shortest Path Problem}

In what follows we will be interested in traffic flowing over the arcs of a network. This makes it necessary to distinguish the direction of the flow, and thus to give a reference direction to the arcs themselves. 
A directed graph or digraph $G=(N, A)$ is a finite nonempty set of nodes $N$ and a collection $A$ of ordered pairs of distinct nodes from $N$; each ordered pair of nodes in $A$ is called a directed arc (or simply arc). Pictorially, a digraph is represented in the same way as a graph, but an arrow is placed on the representation of the arc, going from the first node of the ordered pair (origin) to the second (destination) (see Figure 2.7). Note in 2.7 that $(2,4)$ and $(4,2)$ are different arcs.

The shortest path problem appears in a surprisingly large number of contexts. If $d_{i j}$ is the cost of using a given link $(i, j)$ in a data network, then the shortest path from $i$ to $\mathrm{m}$ is the minimum cost route over which to send data. Thus, if the cost of a link equals the average packet delay to cross the link, the minimum cost route is also a minimum delay route. Unfortunately, in a data network, the average link delay depends on the traffic load carried by the link, which in turn depends on the routes selected by the routing algorithm. Because of this feedback effect, the minimum average delay routing problem is more complex than just solving a shortest path problem, however, the shortest path problem is still an integral part of all the formulations of the routing problem to be considered. As another example, if $P_{i j}$ is the probability that a given link $(i, j)$ in a network is usable and each link is usable independently of all the other links, then finding the shortest path between $\mathrm{i}$ and $\mathrm{m}$ with link distances $\left(-\ln P_{i j}\right)$ is equivalent to finding the most reliable path from $i$ to $m$ [3].

Another application of the shortest path is in PERT (Pilot Emissions Reduction Trading) networks used by organizations to monitor the progress of large projects [3]. The nodes of the network correspond to subtasks, and a link from subtask $i$ to $j$ indicates that the completion of task $j$ depends on the completion of $i$. If $t_{i j}$ is the time required to complete $j$ after $i$ is completed, the distance for $(i, j)$ is taken as $d_{i j}=-t_{i j}$. The shortest path from project start to finish is then the most time-consuming path required for 
completion of the project, and the shortest path indicates the critical subtasks for which delays would delay the entire project. Yet another example is that of discrete dynamic programming problems, which can be viewed as shortest path problems [6]. Finally, many more complex graph-theoretic problems require the solution of shortest path problems as subproblems.

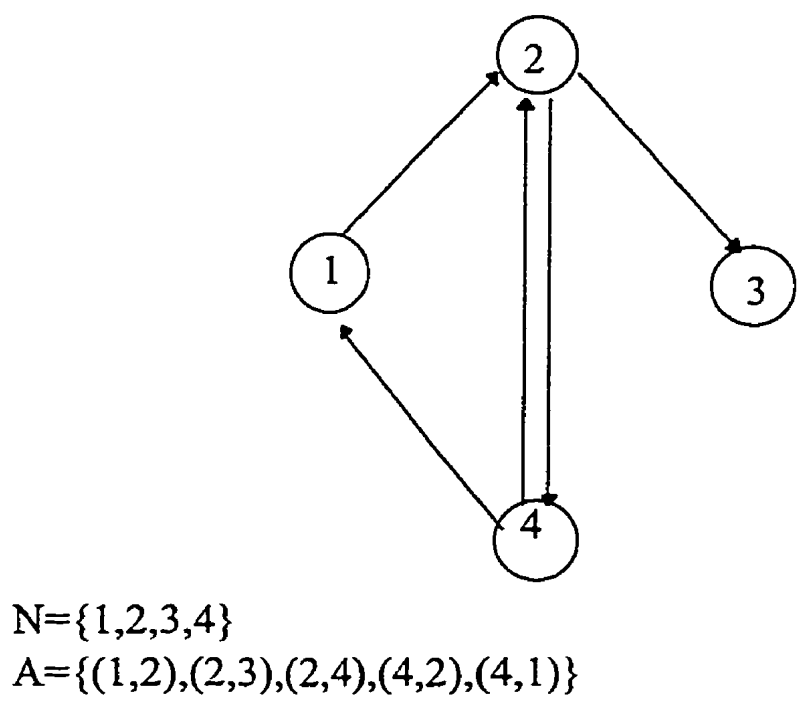

Figure 2.7 Representation of a directed graph

\subsection{Shortest Path Algorithms}

In the following, we consider three standard algorithms for the shortest path problem: the Bellman-Ford algorithm, the Dijkstra algorithm, and Floyd-Warshall algorithm. The first two algorithms find the shortest paths from all nodes to a given destination node, and the third algorithm finds the shortest paths from all nodes to all other nodes. Note that the problem of finding shortest paths from a given origin node to all other nodes is equivalent to the problem of finding all shortest paths to a given destination node; one version of the problem can be obtained from the other simply by reversing the direction of each link while keeping its length unchanged. 


\subsubsection{The Bellman-Ford Algorithm [3]}

Suppose that node 1 is the "destination" node and consider the problem of finding a shortest path from every node to node 1 . Assume that there exists at least one path from every node to the destination. Let us denote $d_{i j}=\infty$ if $(i, j)$ is not an arc of the graph. Using this convention we can assume without loss of generality that there is an arc between every pair of nodes, since walks and paths consisting of true network arcs are the only ones with length less than $\infty$.

A shortest walk from a given node $i$ to node 1 , subject to the constraint that the walk contains at most $h$ arcs and goes through node 1 only once, is referred to as a shortest $(\leq h)$ walk and its length is denoted by $D_{i}^{h}$. Note that such a walk may not be a path, that is, it may contain repeated nodes. By convention, we take

$$
D_{1}^{h}=0 \quad \text { for all } h
$$

and

$$
D_{i}^{h+1}=\min _{j}\left[d_{i j}+D_{j}^{h}\right], \quad \text { for all } i \neq 1
$$

This is the Bellman-Ford algorithm [3], illustrated in Figure 2.8. The Bellman-Ford algorithm first finds the one-arc shortest walk lengths, then finds the two-arc shortest walk lengths, and so forth. The algorithm terminates after $h$ iterations if

$$
D_{i}^{h}=D_{i}^{h-1}, \text { for all } i
$$

The following proposition provides the main results [3].

Proposition. Consider the Bellman-Ford algorithm (2.1) with the initial conditions $D_{i}^{0}=\infty$ for all $i \neq 1$. Then: 
(a)- The scalars $D_{i}^{h}$ generated by the algorithm are equal to the shortest $(\leq h)$ walk lengths from node $i$ to node 1 .

(b)- The algorithm terminates after a finite number of iterations if and only if all cycles not containing node 1 have nonnegative length. Furthermore, if the algorithm terminates, it does so after at most $h \leq N$ iterations, and at termination, $D_{i}^{h}$ is the shortest path length from $i$ to 1 .

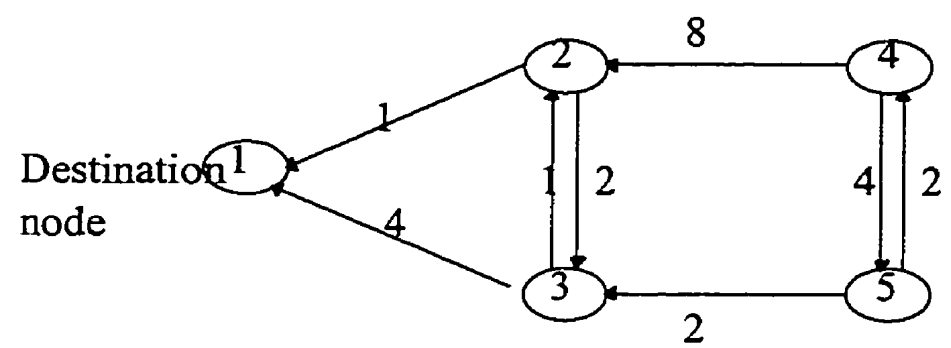

Shortest path problem, arc length as indicate

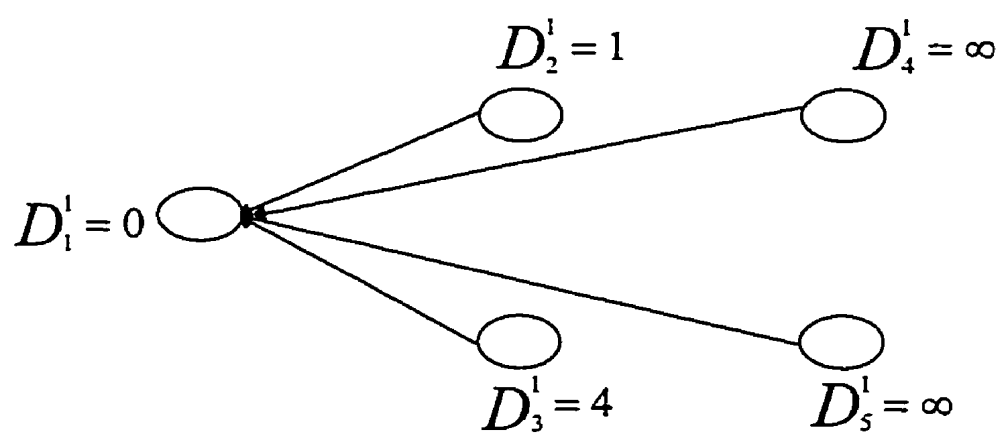

Shortest paths using at most 1 arc 


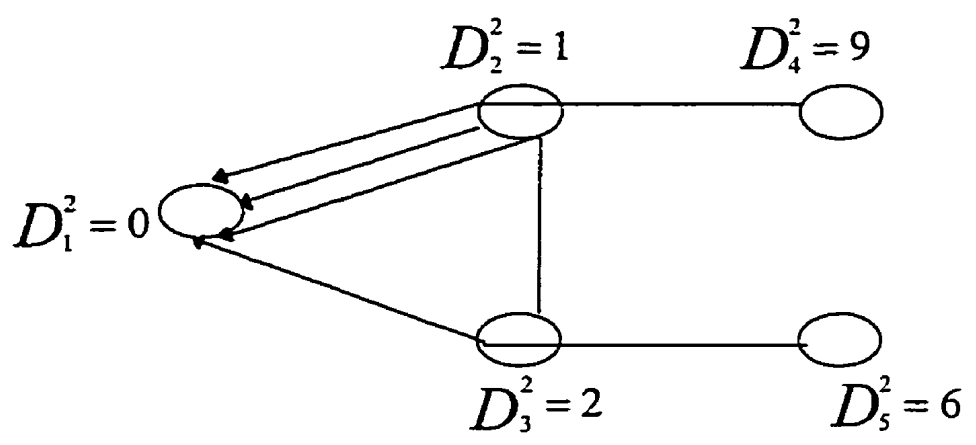

Shortest paths using at most 2 arcs

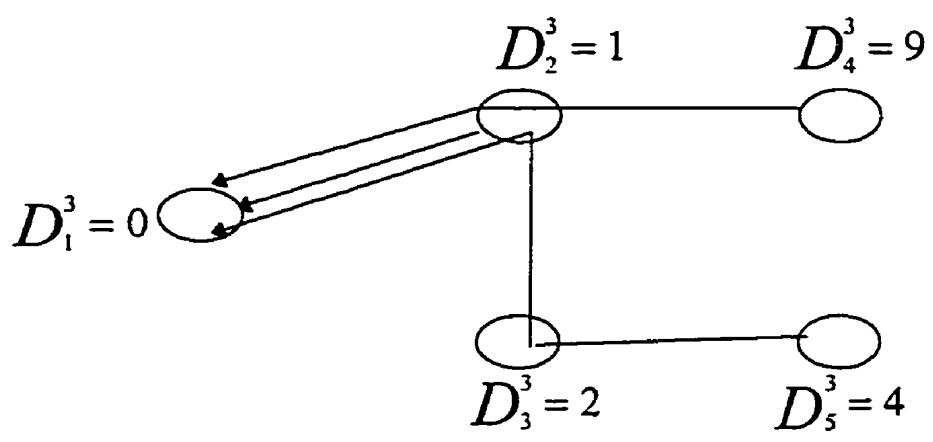

Shortest paths using at most 3 arcs

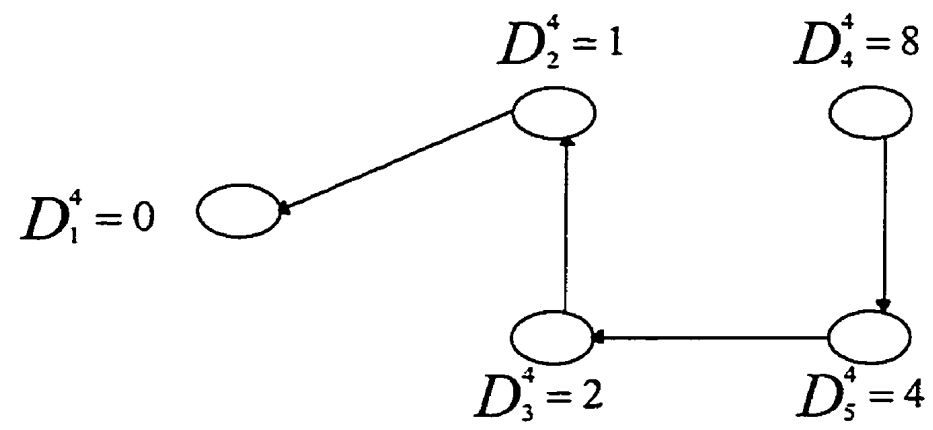

Final tree of shortest paths

Figure 2.8 Successive iterations of the Bellman-Ford method. In this example, the shortest $(\leq h)$ walks are paths because all arc lengths are positive and therefore all cycles have positive length. The shortest paths are found after $N-1$ iterations. Which is equal to 4 in this example. 


\subsubsection{Dijkstra's Algorithm [3]}

This algorithm requires that all arc lengths are nonnegative (furtunately, the case for most data network applications). Its worst case computational requirements are considerably less than those of the Bellman-Ford algorithm. The general idea is to find the shortest path order of increasing path length. The shortest of the shortest paths to node 1 must be the single-arc path from the closest neighbor of node 1 , since any multiple-arc path cannot be shorter than the first arc length because of the nonnegative-length assumption. The next shortest of the shortest paths must either be the single-arc path from the next closest neighbor of 1 or the shortest two-arc path through the previously chosen node, and so on. To formalize this procedure into an algorithm, each node $i$ is viewed as being labeled with an estimate $D_{i}$ of the shortest path length to node 1 . When the estimate becomes certain, we regard the node as being permanently labeled and keep track of this with a set $P$ of permanently labeled nodes. The node added to $P$ at each step will be the closest to node 1 out of those that are not yet in $P$. Figure 2.9 illustrates the main idea. The detailed algorithm is as follows [3]:

Initially, $P=\{1\}, D_{1}=0$, and $D_{j}=d_{j 1}$ for $j \neq 1$.

Step 1: (Find the next closest node.) $i \in P$ such that

$$
D_{i}=\min _{j \in P} D_{j}
$$

set $P=P \bigcup\{i\}$. If $\mathrm{P}$ contains all nodes, then stop; the algorithm is complete.

Step 2: (Updating of labels) For all $j \notin P$ set

$$
D_{j}=\min \left[D_{j}, d_{j i}+D_{i}\right]
$$

Go to step 1 .

To see why the algorithm works, we must interpret the estimates $D_{j}$. We claim that at the beginning of each step:

(a) $D_{i} \leq D_{j}$ for all $i \in P$ and $j \notin P$. 
(b) $D_{j}$ is, for each node $j$, the shortest distance from $j$ to 1 using paths with all nodes except possibly $j$ belonging to the set $P$.

Indeed, condition (a) is satisfied initially, and since $d_{j i} \geq 0$ and $D_{i}=\min _{j \notin P} D_{j}$, it is preserved by the formula $D_{j}=\min \left[D_{j}, d_{j i}+D_{i}\right]$ for all $j \notin P$, in step 2 . We show condition (b) by induction. It holds initially. Suppose that it holds at the beginning of some step 1 , let $i$ be the node added to $P$ at that step, and let $D_{k}$ be the label of each node $k$ at the beginning of that step. Then condition (b) holds for $j=i$ by the induction hypothesis. It is also seen to hold for all $j \in P$, in view of condition (a) and the induction hypothesis. Finally, for a node $j \notin P \bigcup\{i\}$, consider a path from $j$ to $i$ which is shortest among those with all nodes except $j$ belonging to the set $P \bigcup\{i\}$, and let $D_{j}^{\prime}$ be the corresponding shortest distance. Such a path must consist of an arc $(j, k)$ for some $k \in P \bigcup\{i\}$, followed by a shortest path from $k$ to 1 with nodes in $P \bigcup\{i\}$. Since we just argued that the length of this $k$ to 1 path is $D_{k}$, we have

$$
D_{j}^{\prime}=\min _{k \in P \cup\{i\}}\left[d_{j k}+D_{k}\right]=\min \left[\min _{k \in P}\left[d_{j k}+D_{k}\right], d_{j i}+D_{i}\right]
$$

Similarly, the induction hypothesis implies that $D_{j}=\min _{k \in P}\left[d_{j k}+D_{k}\right]$, so we obtain $D_{j}^{\prime}=\min \left[D_{j}, d_{j i}+D_{i}\right]$. Thus in step $2, D_{j}$ is set to the shortest distance $D_{j}^{\prime}$ from $j$ to 1 using paths with all nodes except $j$ belonging to $P \bigcup\{i\}$. The induction proof of condition (b) is thus complete.

We now note that a new node is added to $P$ with each iteration, so the algorithm terminates after $N-1$ iterations, with $P$ containing all nodes. By condition (b), $D_{j}$ is then equal to the shortest distance from $j$ to 1 . 
To estimate the computation required by Dijkstra's algorithm, note that there are $N-1$ iterations and the number of operations per iteration is proportional to $N$. therefore, in the worst case the computation is $O\left(N^{2}\right)[1]$, comparing favorable with the worst-case estimate $O\left(N^{3}\right)$ of the Bellman-Ford algorithm [1]; in fact, with proper implementation the worst-case computational requirements for Dijkstra's algorithm can be reduced considerably [7].

Shortest path from the $(\mathrm{k}+1)$ st closest node must pass exclusively through nodes in $\mathrm{P}$

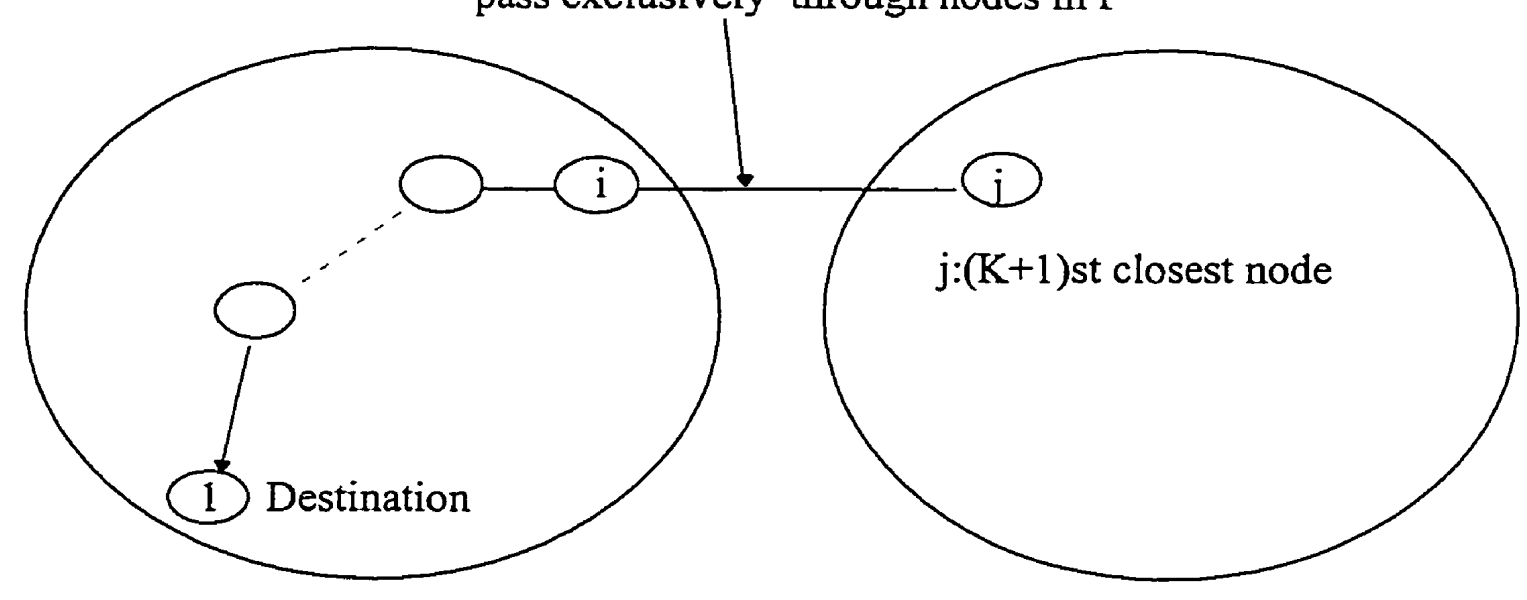

Set $\mathrm{P}$ of $\mathrm{K}$ closest nodes to node 1

Complement of $\mathrm{P}$

Figure 2.9 Basic idea of Dijkstra's algorithm. At the $K^{\text {th }}$ step we have the set $\mathrm{P}$ of the $\mathrm{k}$ closest nodes to node 1 as well as the shortest distance $D_{i}$ from each node $\mathrm{i}$ in $\mathrm{P}$ to node 1. Of all paths connecting some node not in $\mathrm{P}$ with node 1 , there is a shortest one that passes exclusively through nodes in $\mathrm{P}$ (since $d_{i j} \geq 0$ ). Therefore, the $(\mathrm{k}+1)$ st closest node and the corresponding shortest distance are obtained by minimizing over $j \notin P$ the quantity $\min _{i \in P}\left\{d_{j i}+D_{i}\right\}$. 
The Dijkstra and Bellman-Ford algorithms are contrasted on an example problem in Figure 2.10.

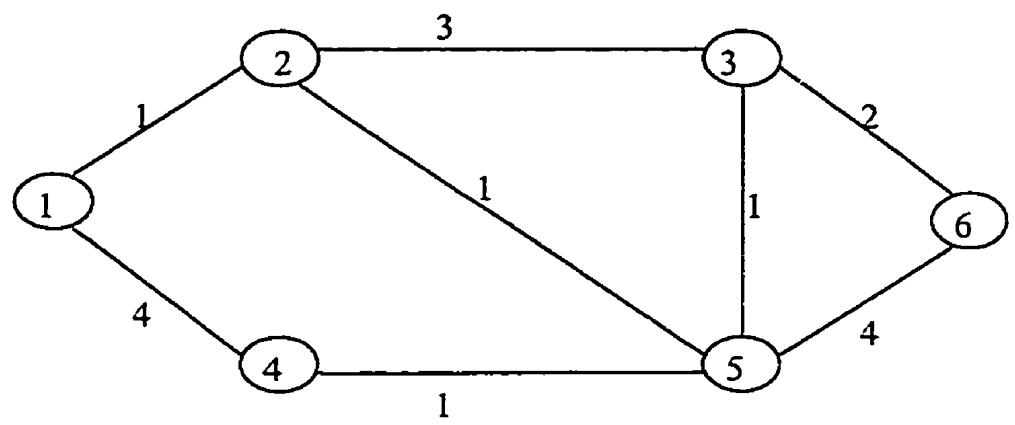

Bellman-Ford
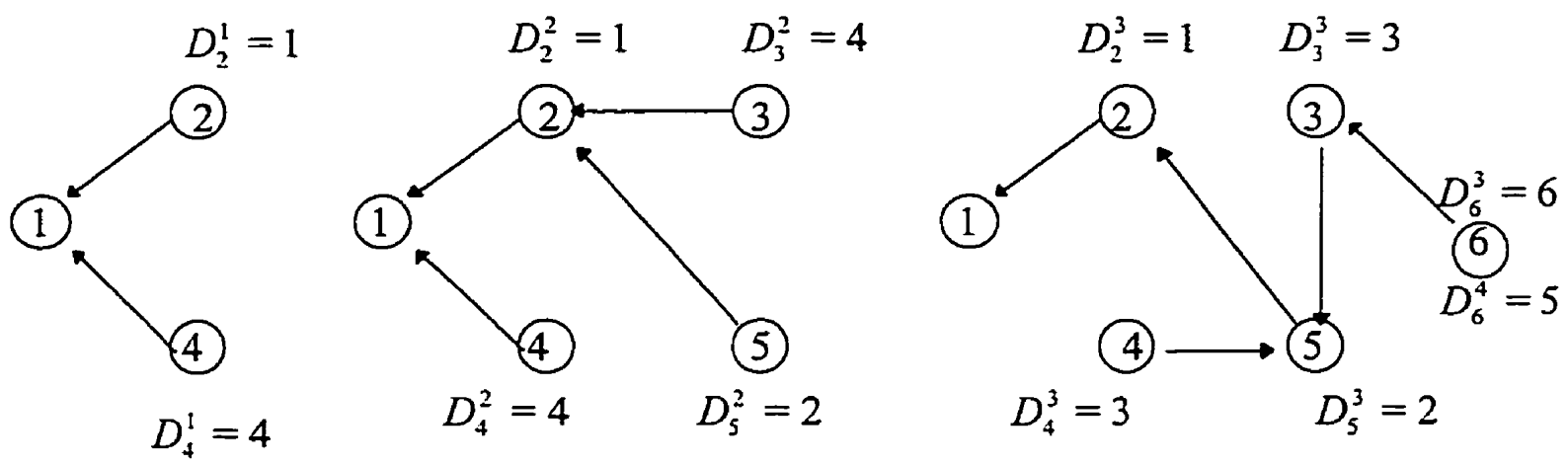
Dijkstra

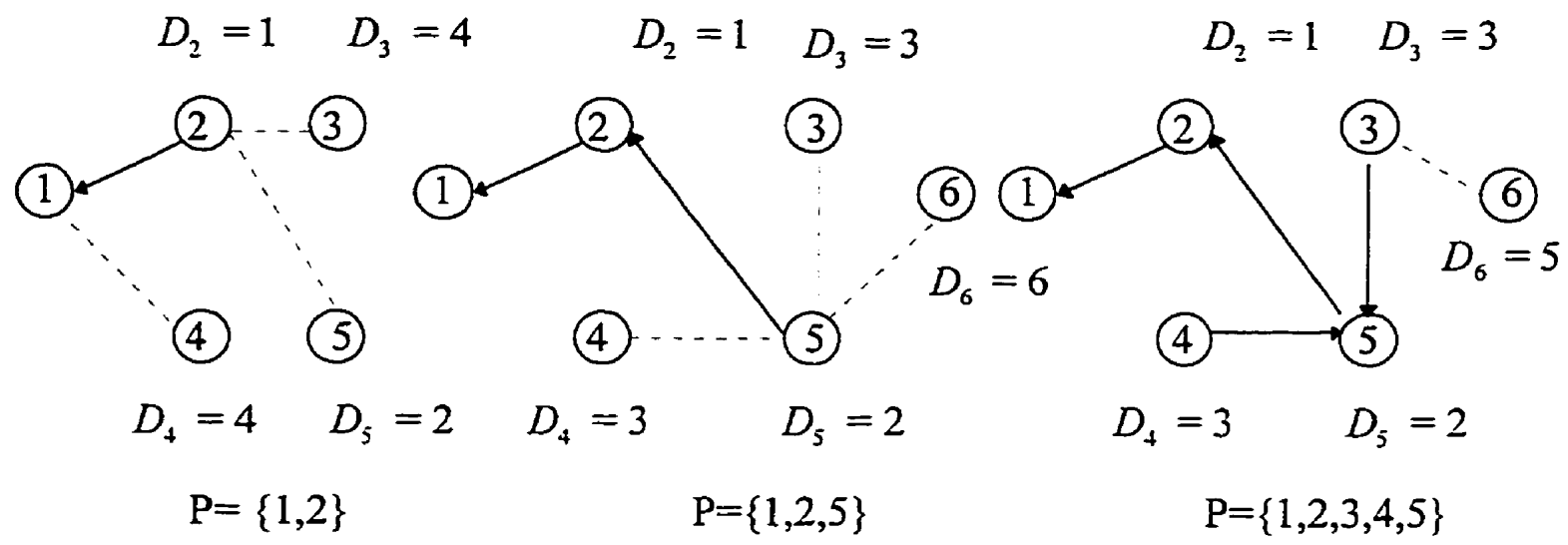

Figure 2.10 Example using the Bellman-Ford and Dijkstra algorithms.

\subsubsection{The Floyd-Warshall Algorithm [3]}

This algorithm, unlike the previous two, finds the shortest paths between all pairs of nodes together. Like the Bellman-Ford algorithm, the arc distances can be positive or negative, but again there can be no negative-length cycles. All three algorithms iterate to find the final solution, but each iterates on something different. The Bellman-Ford algorithm iterates on the number of arcs in a path, the Dijkstra's algorithm iterates on the length of the path, and finally, the Floyd-Warshall algorithm iterates on the set of nodes that are allowed as intermediate nodes on the paths. The Floyd-Warshall algorithm starts like both other algorithms with single arc distances (i.e. no intermediate nodes) as starting estimates of shortest path length. It then calculates shortest paths under the constraint that only node lcan be used as an intermediate node, and then with the constraint that only nodes 1 and 2 can be used, and so forth. 
To state the algorithm more precisely, let $D_{i j}^{n}$ be the shortest path length from node $i$ to $j$ with the constraint that only nodes $1,2, \ldots ., n$ can be used as intermediate nodes on paths. The algorithm then is as follows [3]: Initially

$$
D_{i j}^{0}=d_{i j}, \quad \text { for all } i, j, i \neq j
$$

For $n=0,1, \ldots, N-1$,

$$
D_{i j}^{n+1}=\min \left[D_{i j}^{n}, D_{i(n+1)}^{n}+D_{(n+1) j}^{n}\right], \text { for all } i \neq j
$$

To see why this works, we use induction. For $n=0$, the initialization clearly gives the shortest path lengths subject to the constraint of no intermediate nodes on paths. Now, suppose that for a given $n, D_{i j}^{n}$ in the algorithm above gives the shortest path lengths using nodes 1 to $n+1$ as intermediate nodes. Then the shortest path length from $i$ to $j$, allowing nodes 1 to $n+1$ intermediate nodes, either contains node $n+1$ on the shortest path, or does not contain node $n+1$. For the first case, the constrained shortest path from $i$ to $j$ goes first from $i$ to $n+1$ and then from $n+1$ to $j$, giving the length in the final term of Equation (2.2). for the second case, the constrained shortest path is the same as the one using nodes 1 to $\mathrm{n}$ as intermediate nodes, yielding the length of the first term in the minimization of Equation (2.2).

Since each of the $N$ steps above must be executed for each pair of nodes, the computation involved in the Floyd-Warshall algorithm is $O\left(N^{3}\right)[1]$, the same as if the Dijkstra's algorithm were repeated for each possible choice of source node.

\subsection{Link Costs}

Should the cost of a link be a fixed number, or should it be a quantity that varies with the utilization of the link? If the cost is a fixed number, should it be set automatically at link 
start-up, by some sort of algorithm in which the bandwidth and delay are measured and a number is calculated that is a function of the measured characteristics? Or should it be left solely to the discretion of the network manager to assign the number, based either on the characteristics of the link or on the knowledge of the expected traffic matrix, so that traffic can be discouraged from using links that are likely to become congested with that matrix?

The alternative to establish a fixed quantity is to have the routers measure the delay on each link and increase the link's cost as its utilization increases, thereby encouraging traffic to seek an alternate, and perhaps less congested, route.

These are important and interesting questions, and they have not been adequately studied. The proponents of having link costs vary with traffic offer two major arguments [1]:

1- Traffic will be routed more optimally if the current traffic conditions are factored into the link costs and thus into the route calculations.

2- Having link costs assigned by network managers requires additional configuration, which is undesirable both because it involves unnecessary work and because it introduces yet another place where human error can create problems.

The proponents of fixed link costs argue that [1]:

I- If the cost of a link is fixed quantity, routing information about the link only needs to be generated if the link goes down or recovers. This is a far less frequent event than changes in the traffic pattern on the link. Thus, with fixed link costs, there will be lot less control traffic, which might more than offset the gain in network capacity that could be realized by using more optimal routes.

2- Between the time when a link changes cost and the time when knowledge of that revised link cost has successfully reached all the routers and they have all completed their 
routing computations, routing is disrupted in the networks, since routers are making routing decisions based on different data. If link costs are frequently changing, the network will more often be in an "unconverged" state, in which routing will be disrupted to some degree, ranging from not at all to globally and severely. When the network is in an unconverged state, looping data packets will consume a significant portion of the network capacity that was perhaps gained by more optimal routing. The proportion of time that the network is in an unconverged state is also an annoyance, since packets might be dropped and even transport layer connections might not survive the disruption. Although "network quality" is an intangible and hard-to-measure factor, it must be taken into account when comparing the schemes.

If fixed costs are used, there is still the question of whether to have the cost assigned by humans or calculated by the routes upon link start-up. And if the cost are humanassigned, another question is whether the costs should be a simple function of the characteristics of the link, such as its bandwidth and delay, or whether the costs should take into account the expected traffic matrix in the network.

The proponents of traffic-matrix-dependent costs argue that network capacity will be increased if costs depend on the traffic matrix and are set as to optimize flow. The opponents argue that attempting to optimize to that degree is a complex task, and it is likely to be done incorrectly, since the operating topology will differ from the topology on which link costs were optimized if new nodes are added to the network or if links or routers are down, and furthermore, the actual matrix might differ from the expected one. Simple link costs in which all equivalent links (all of the same delay and bandwidth) are assigned the same value are safer.

An additional approach for optimizing traffic flow in a network that has not been implemented anywhere is to have congestion information transmitted around the network 
in a manner that does not change the link costs but is only used by routers as advice for choosing among equivalent paths. This has the disadvantage of introducing more control traffic (the congestion information) but may enhance network capacity [1].

My opinion on all this is that attempting to increase network capacity a very small amount through complex and costly algorithms is a bad idea. I believe that the difference in network capacity between the simplest form of link assignment (fixed values depending on the delay and total bandwidth of the link) and "perfect routing" (in which a deity routes every packet optimally, taking into account traffic currently in the network as well as traffic that will enter the network in the near future) is not very great. The difference between simple routing and the more optimal routing that can be gained by constantly monitoring delays on each link and adding control traffic to keep all the other routers informed is probably negligible, especially when the added bandwidth consumed by the routing information is taken into account.

If fixed costs are used, I do not feel that fancy "traffic matrix" algorithms that are run on the assumed topology in order to optimize traffic flow are a good idea, since I believe that the assumptions under which the numbers are generated are almost guaranteed to be incorrect (topology will evolve as nodes and links are added; topology will change as nodes and links fail; traffic patterns are not necessarily predictable). I do not believe that the percentage of network capacity that could theoretically be gained is large enough to warrant the complexity and risk.

Having routers measure the characteristics of links and set the costs at link startup seems like a good idea. It eliminates the possibility of human error, makes the network more "plug and play," and does not add any control traffic or increase the time during which the network is unconverged. Nobody has implemented such a scheme, however. 


\section{Chapter 3}

\section{Neural Networks}

\subsection{What are Neural Networks, and What are They Good for?}

In spite of the fact that modern digital computers have made leaps in both speed and processing power, there are certain tasks that may not be performed sufficiently well by computers due to the complexities associated with programming a computer to perform the tasks. Such include optimization, visual pattern recognition, and classification. Neural networks can solve these problems more easily than conventional computers while sophisticated scientific and mathematical problems are ideally suited to computers.

The 1980's witnessed a resurgence of interest in neural networks [8]. This new interest is due to the development of new network topologies, algorithms, and new analog VLSI implementations $[4,9,10]$. A neural network is a parallel, distributed information processing structure consisting of many processing elements interconnected via weighted connections. The idea of neural network was inspired by the structure of the human brain and by admiration of the computing power of the brain. A processing element in a neural network is called an artificial neuron, and a generic structure is shown in Figure 3.1. The input signals arriving at the neuron are supplied to the transfer function. The output of the neuron is determined by the weighted sum of inputs and the transfer function. A neural network consists of large numbers of this simple processing elements that are connected to each other and operating in parallel. It must be mentioned that a neural network must be distinguished from a parallel computer. Whereas the fundamental problem with 
conventional parallelism is how to program the multiprocessors; with neural networks it is how to determine the connections between processing elements [8].

One of the most prevalent uses of neural networks is optimization. Optimization is a technique for solving a problem by casting it into a mathematical equation that, when either maximized or minimized, solves the problem. Typical examples of problems approached by an optimization technique include scheduling, routing, and source allocation. The neural optimization approach casts the optimization problem into the form of an energy function that describes the dynamics of a neural systems. If the neural network dynamics are such that the network will always seek a stable state when the energy function is at a minimum, then the final values of the processing elements represent a solution.

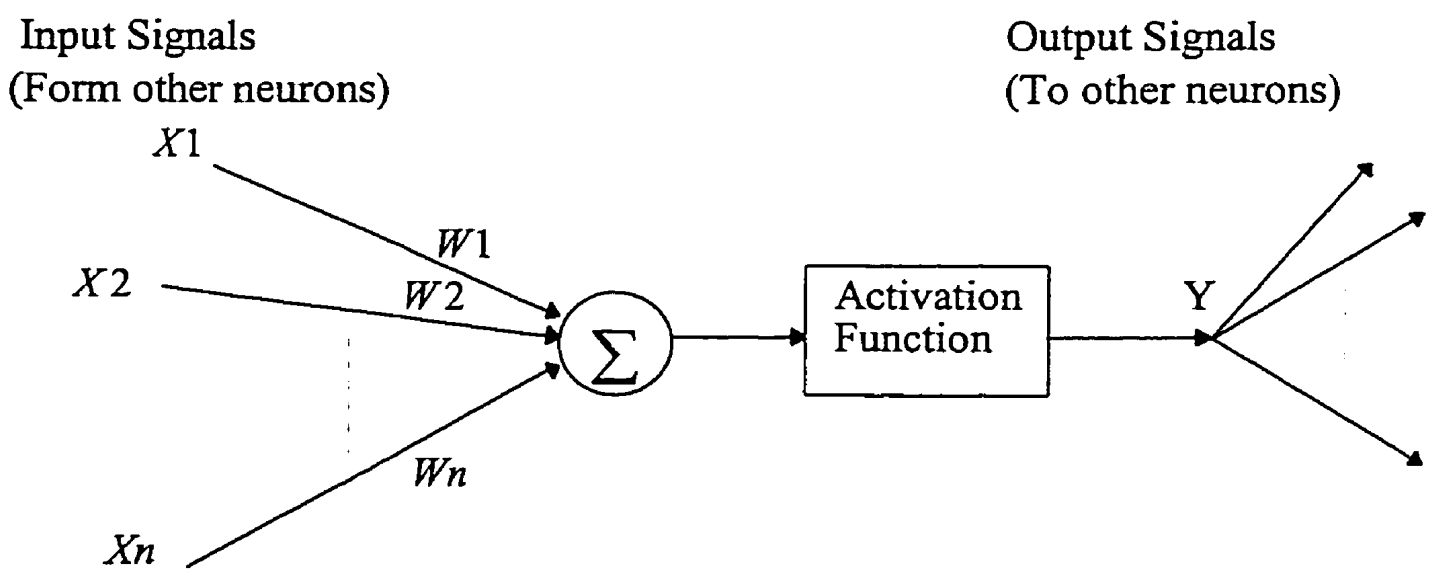

Figure 3.1 A Generic Processing Element

\subsection{Neural Networks for Optimization}

Neural networks have been used to solve a variety of constrained optimization problems $[2,11,12,13,14,15]$. Due to their massive parallelism and fast network convergence to optimal solutions, they eliminate the time bottlenecks that usually arise in most sequential 
algorithms. Solving optimization problems requires minimization of some cost functions subject to a set of constraints. These cost functions are known as energy functions, and the neural network will produce good solutions by minimizing an energy function.

Many people contributed to neurocomputing's renaissance. However, the efforts of John Hopfield in the years 1982 to 1986 were unique in their effectiveness. Since Hopfield and Tank [2] proposed an artificial neural network in 1985 for solving difficult optimization problems like the Traveling Salesman Problem (TSP), many researchers have applied this approach to a number of constrained optimization problems. The circuit diagram of a Hopfield network is shown in Figure 3.2.

In a Hopfield neural network each processing element has an external input and has weighted connections from other neurons. The connectivity between neurons is represented by an $n \times n$ weight matrix $W=\left[W_{i j}\right]$ with the restriction that $W_{i j}=W_{j i}$, where $W_{i j}=\frac{1}{R_{i j}}$. A connection from a positive output is known as excitatory, and a connection from a negative output is called an inhibitory connection. Let $U_{i}$ and $V_{i}$ denote the network input to $i^{\text {th }}$ neuron and its output respectively, then the network input to ith neuron is

$$
U_{i}=\sum_{j=1}^{n} W_{i j} V_{j}+I_{i}
$$

where $I_{i}$ is the external input.

The output of a neuron is determined by the network input and the transfer function of the neuron. Figure 3.3 shows the input node of the $i^{i h}$ neuron. 


\section{External Inputs}

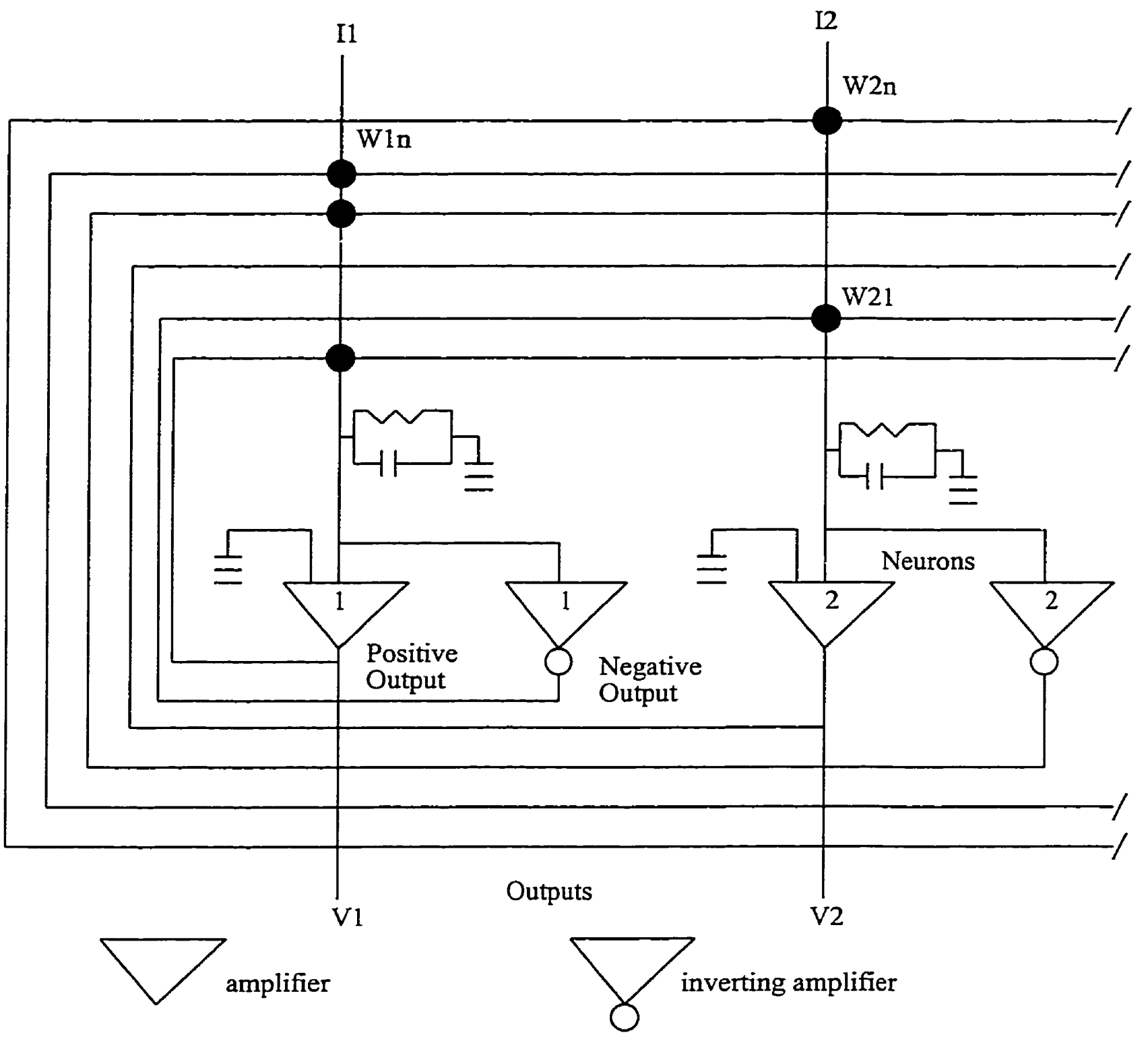

resistor in Wij network

Figure 3.2 Hopfield Neural Network 


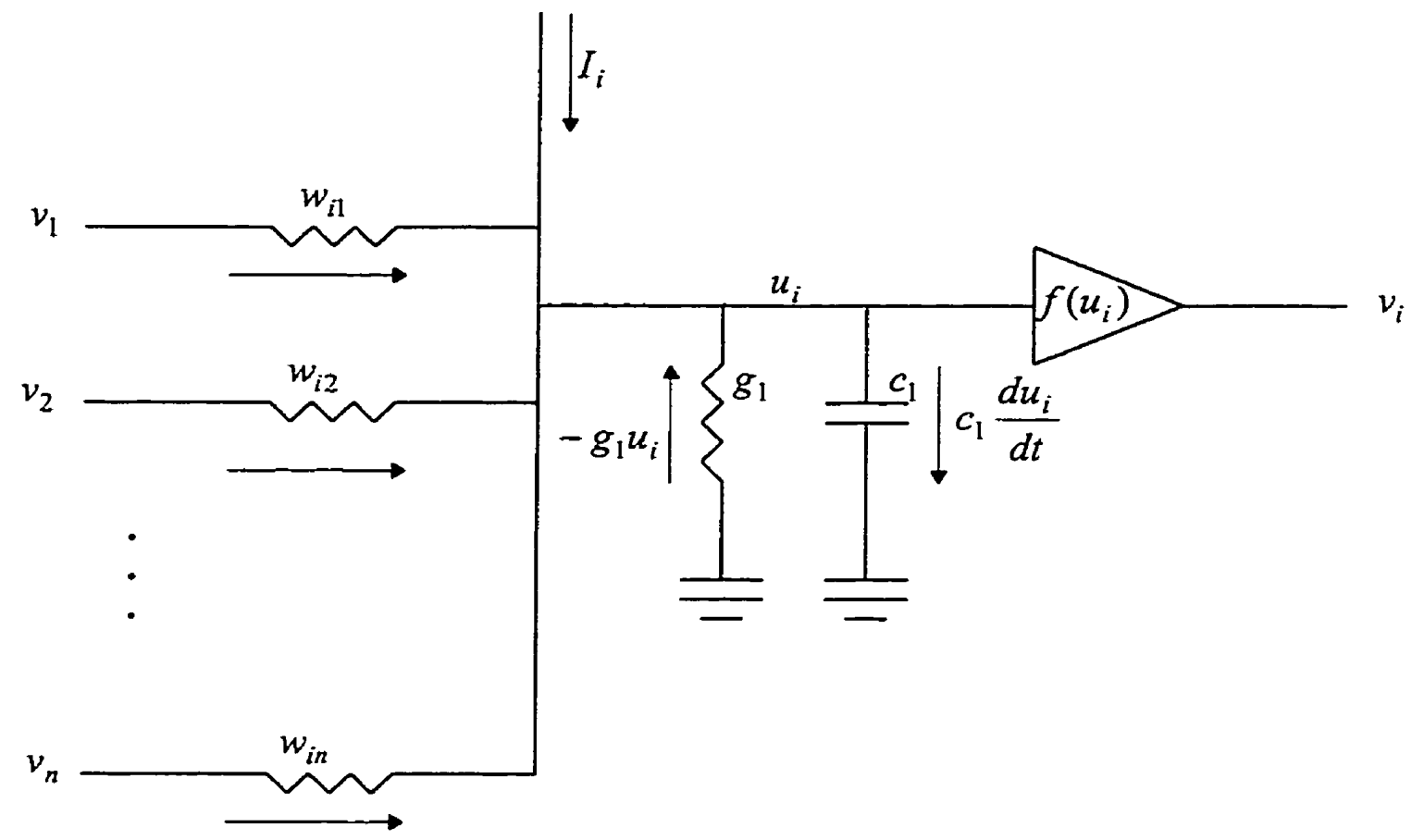

Figure 3.3 Input node of the ith neuron

The transfer function of the Hopfield module is given by

$$
\begin{aligned}
V_{i} & =f\left(\lambda U_{i}\right) \\
& =\frac{1}{2}\left\{1+\tanh \left(\lambda U_{i}\right)\right\}
\end{aligned}
$$

where $\lambda$ is a constant called the gain parameter.

This equation represents a continuos, nondecreasing, and differentiable function called a sigmoid function. 
In addition, as shown in Figures 3.2 and 3.3, each neuron receives an external current (known also as bias) $I_{i}$, which could represent actual data provided by the user to the neural network [17]. From Figure 3.3 the dynamics of the Hopfield network can be described by

$$
C_{i} \frac{d U_{i}}{d t}=\sum_{j=1}^{N} W_{i j} \cdot V_{j}-\frac{U_{i}}{R}+I_{i}
$$

Dividing by $C$ and redefining $\frac{W_{i j}}{C}$ and $\frac{I_{i}}{C}$ as $W_{i j}$ and $I_{i}$, the dynamics of the network become:

$$
\frac{d U_{i}}{d t}=-\frac{U_{i}}{\tau}+\sum_{j=1}^{N} W_{i j} \cdot V_{j}+I_{i}
$$

where $\tau=R C$ is the circuit's time constant, and $N$ is the number of neurons in the network.

Hopfield [17] has shown that the dynamics of the neurons (3.3) follow a gradient descent of the quadratic energy function

$$
E=-\frac{1}{2} \sum_{i=1}^{N} \sum_{j=1}^{N} W_{i j} V_{i} V_{j}-\sum_{i=1}^{N} I_{i} V_{i}
$$

Hopfield [17] has also shown that while the state of the neural network evolves inside the N-dimensional hypercube defined by $V_{i} \in\{0,1\}$, the minima of the energy function (3.4) occur at the $2^{N}$ corners of this space, only if $\lambda_{i}=\infty$. 
In terms of the energy function (3.4), the dynamics of the ith neuron are described by

$$
\frac{d U_{i}}{d t}=-\frac{U_{i}}{\tau}-\frac{\partial E}{\partial V_{i}}
$$




\section{Chapter 4}

\section{Neural Networks and Routing}

\subsection{Introduction}

Routing methods must evolve to maximize network utilization under normal conditions and adapt to and minimize the effects of abnormal network conditions. Conventional routing approaches are superior to older methods, but still have disadvantages in terms of speed, flexibility, and required memory. By utilizing the adaptive properties of neural networks, advanced network routing methods could be created without suffering the disadvantages of conventional algorithms.

Based on the review of data communications, routing theory, and existing neural network applications, the list of attributes of a preferred network routing system using neural networks can be defined. These are:

1. the method must perform routing decisions quickly,

2. the method should be distributed to provide the fast decision times,

3. the routing method should utilize information provided by adjacent nodes for performing routing decisions, and

4. a neural network implementation should be scalable.

The approach to the problem is based on a solution proposed for the traveling salesman problem (TSP) using Hopfield neural network as given in [2]. The routing problem is considerably similar to the TSP problem in that it is concerned with finding an optimal path which travels between a source and a destination node (city). 


\subsection{Routing Using Hopfield Neural Network}

The use of neural networks to find the shortest path between a given source-destination pair was initiated by Rauch and Winarske [13]. They proposed a neural network architecture arranged in a two-dimensional array of size $n \times m$, where $m$ is the number of nodes forming the path. The output $V_{x i}$ of the neuron at location $(x, i)$ is defined as follows:

$$
\begin{aligned}
V_{x i} & =1 \text { if node } x \text { is the } i \text { th node to be visited in the path } \\
& =0 \text { otherwise. }
\end{aligned}
$$

One obvious limitation of the above representation is that it requires a priori knowledge of the number of nodes forming the shortest path, however this number is usually unknown. In [13], $m$ is fixed to the minimum number of nodes forming the path. It is obvious then that the Rauch and Winarske algorithm finds the shortest path only among those $m$ node paths. Therefore their algorithm gives only a suboptimal solution to the problem; after all the shortest path may consist of more than $m$ nodes. To correct this shortcoming, Zhang and Thompoulos [18] extended $m$ to the total number of nodes in the network, which is also the maximum number of nodes the path may consist of. They assigned zero-cost self-loops connecting each node to itself and very large costs to nonexisting links. In this way a self loop can be included in the path without increasing the path cost. For a given source-destination path, they also fixed the state of all neurons located at the first and last column, while allowing the remaining neurons to evolve so as to minimize the following energy function:

$$
E=\frac{A}{2} \sum_{k=1}^{n-1} \sum_{i=1}^{n} \sum_{j=1}^{n} V_{i k} C_{i j} V_{j k+1}+\frac{B}{2} \sum_{k=1}^{n} \sum_{i=1}^{n} \sum_{j=1}^{n} V_{i k} V_{j k}+\frac{C}{2}\left(\sum_{i=1}^{n} \sum_{j=1}^{n} V_{i j}-n\right)^{2}
$$


In the above the $A$ term represents the total cost of the path, while the $B$ and $C$ terms are constraints introduced to force the neural network to converge to a valid path. The B term is minimized if each column contains at most a single 1 , which corresponds to at most one node visited at a time. The $\mathrm{C}$ term ensures that there will be exactly $n \mathrm{l}$ 's in the final solution. When combined together, the $B$ and $C$ term ensure that each column will have exactly a single 1 .

Although the neural network formulation (4.2) is optimal, it still has significant drawbacks. Since each neuron belonging to the first and last column has a fixed output voltage, the neural network is then designed to find the shortest path between only a given source-destination pair. To find a route between another pair, the neural configuration has to be changed. More importantly, since the cost term in the energy function (4.2) is quadratic, the connection weights among the neurons depend on link costs. In practice the link costs in a communication network are usually time varying. Consequently Zhang and Thompoulos' neural network [18] will not be suitable for use in routing since the resistances of the synaptic connections must be changed continuously in order to adapt to changes in link costs. In what follows a new approach to the problem, also based on the Hopfield model, is presented.

\subsection{Problem Formulation}

To formulate the routing problem in terms of Hopfield neural network, a suitable representation scheme must be found so that the shortest path can be decoded from the final stable state of the neural network. The proposed model is organized in an $(n \times n)$ matrix, with all diagonal elements removed, since they are not needed. Each element in the matrix is represented by a neuron which is described by double indices $(x, i)$, where the row subscript $x$ and the column subscript $i$ denote node numbers. Therefore the computational network requires $n(n-1)$ neurons, and a neuron at location $(x, i)$ is characterized by its output $V_{x i}$, defined as follows: 


$$
\begin{aligned}
V_{x i} & =1 \text { if the link from node } x \text { to node } i \text { is in the shortest path } \\
& =0 \text { otherwise. }
\end{aligned}
$$

Also a matrix $\Gamma=\left[P_{x i}\right]$ is defined for the link connection as follows

$$
P_{x i}=1 \text { if the link from node } x \text { to node } i \text { does not exist }
$$$$
=0 \text { otherwise. }
$$

In addition the cost of a link from node $x$ to node $i$ is denoted by $C_{x i}$, a finite real positive number. For nonexisting links this cost is assumed to be zero.

\subsection{The Energy Function and The Motion Equation}

In order to solve the routing problem, using a Hopfield neural network, we first have to define an energy function whose minimization process drives the neural network into its lowest energy state. This stable state shall correspond to the shortest path solution. The energy function must favor states that correspond to valid paths between the specified source-destination pair. Among these valid paths it must also favor the one which has the lowest cost. In this thesis we have used the same energy function given in [21] which satisfies such requirements it is constructed as follows:

1. The first term minimizes the total cost of a path by taking into account the cost of the existing links.

$$
E_{\mathrm{l}}=\frac{T_{1}}{2} \sum_{\substack{x=1 \\ \text { ind } \\ i \neq x \\(x, i) \neq(d, s)}}^{n} C_{x i} V_{x i}
$$

2. The second term prevents nonexisting links from being included in the chosen path. 


$$
E_{2}=\frac{T_{2}}{2} \sum_{\substack{x=1 \\ \text { i } \\ i \neq 1 \\(x, i) \neq(d, s)}}^{n} P_{x i} V_{x i}
$$

3. The third term makes sure that if a node has been entered it will also be exited by a path

$$
E_{3}=\frac{T_{3}}{2} \sum_{x=1}^{n}\left(\sum_{\substack{i=1 \\ i \neq x}}^{n} V_{x i}-\sum_{\substack{i=1 \\ i \neq x}}^{n} V_{i x}\right)^{2}
$$

this term is zero if the constraints are satisfied (meaning if for every node in the routing solution), and the number of incoming direction links equals the number of outgoing direction links.

4. The fourth term pushes the state of the neural network to converge to one of the $2^{n}$ corners of the hypercube, defined by $V_{x i} \in\{0,1\}$.

$$
E_{4}=\frac{T_{4}}{2} \sum_{i=1}^{n} \sum_{\substack{x=1 \\ x \neq i}}^{n} V_{x i}\left(1-V_{x i}\right)
$$

this term is zero if the constraints are satisfied.

5. The last term introduced to make sure that we have a loop starts and ends at the source node

$$
E_{5}=\frac{T_{5}}{2}\left(1-V_{d s}\right)
$$

This term is zero when the output of the neuron $(d, s)$ settles to 1 . Although the link from the source to the destination is not part of the solution, it is introduced to enforce the construction of a path, which must originate at the source and terminate at the destination. This makes sure that the final solution contains the link from the destination to the source and therefore both the source and the destination nodes will be in the final solution. 
Finally, the total energy function of the network is :

$$
\begin{aligned}
& E=E_{1}+E_{2}+E_{3}+E_{4}+E_{5} \\
& E=\frac{T_{1}}{2} \sum_{\substack { x=1 \\
\begin{subarray}{c}{i=1 \\
i \neq x{ x = 1 \\
\begin{subarray} { c } { i = 1 \\
i \neq x } }\end{subarray}}^{n} C_{x i} V_{x i}+\frac{T_{2}}{2} \sum_{\substack { x=1 \\
\begin{subarray}{c}{i=1 \\
i \neq x{ x = 1 \\
\begin{subarray} { c } { i = 1 \\
i \neq x } }\end{subarray}}^{n} P_{x i} V_{x i}+\frac{T_{3}}{2} \sum_{\substack { x=1 \\
\begin{subarray}{c}{i \neq 1 \\
i \neq x{ x = 1 \\
\begin{subarray} { c } { i \neq 1 \\
i \neq x } }\end{subarray}}^{n}\left(\sum_{\substack{i=1 \\
i \neq x}}^{n} V_{x i}-\sum^{n} V_{i x}\right)^{2} \\
& (x, i) \neq(d, s) \quad(x, i) \neq(d, s) \\
& +\frac{T_{4}}{2} \sum_{i=1}^{n} \sum_{x=1}^{n} V_{x i}\left(1-V_{x i}\right)+\frac{T_{5}}{2}\left(1-V_{d s}\right) \\
& x \neq i
\end{aligned}
$$

where $T_{1}, T_{2}, T_{3}, T_{4}$ and $T_{5}$ are positive constants.

Thus the final solution will always be a loop, with nodes source and destination included. This loop consists of two parts; a directed path from the source to the destination and a link from the destination to the source. If there are no zero cost loops in the network, then the $T_{\mathrm{I}}$ and $T_{3}$ terms will ensure that there will be at most a single 1 at each row and at each column. This guarantees that there will be one to one relationship between the paths and the neural network representations. To illustrate this consider the example depicted in Figure 4.1. For the network shown in Figure 4.1(a), let us assume that the shortest path is $P^{s d}=(1,2,5,6)$. The corresponding links which are to be included in the final solution are shown in Figure 4.1(b). These links form a loop, thereafter referred to as a primary loop. Correspondingly, the neuron output will be represented as shown in Figure 4.1(c), where each node included in the shortest path has a single 1 in its corresponding row/column. Note that since nodes 3 and 4 are not part of the shortest path, they have all zero entries in their corresponding rows/columns. 


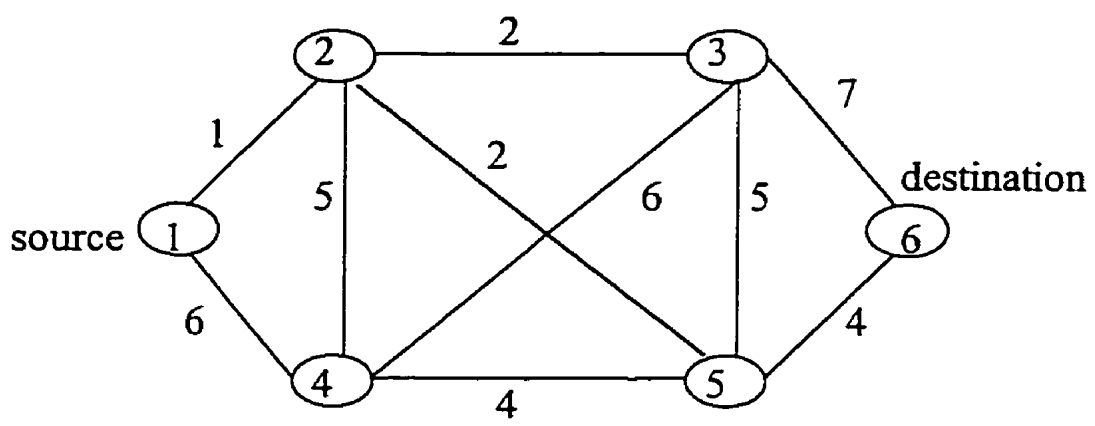

(a)

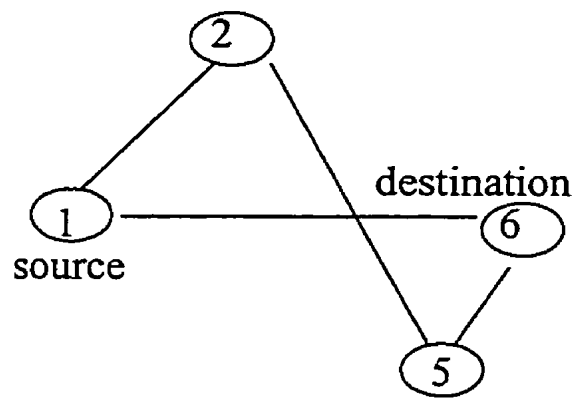

(b) 


\begin{tabular}{|l|l|l|l|l|l|l|}
\hline & 1 & 2 & 3 & 4 & 5 & 6 \\
\hline 1 & 0 & 1 & 0 & 0 & 0 & 0 \\
\hline 2 & 0 & 0 & 0 & 0 & 1 & 0 \\
\hline 3 & 0 & 0 & 0 & 0 & 0 & 0 \\
\hline 4 & 0 & 0 & 0 & 0 & 0 & 0 \\
\hline 5 & 0 & 0 & 0 & 0 & 0 & 1 \\
\hline 6 & 1 & 0 & 0 & 0 & 0 & 0 \\
\hline
\end{tabular}

(c)

Figure 4.1 An illustrative example. (a) The original network. (b) The primary loop forming the shortest path. (c) The neural network output representation.

\subsection{The Weight Matrix and The Biases}

The most important task at hand is to find an appropriate connection weight matrix. It is constructed taking into account the requirement that nonvalid paths should be prevented and valid paths should be preferred. A consideration in this regard is that, for example, no two neurons in the same row (column) should fire during the same cycle of operation of the network.

In this context, the Kronecker delta function is used to facilitate a simple notation. The Kronecker delta function has two arguments, which are given usually as subscripts of the symbol $\delta$. By definition, $\delta_{a b}$ has value 1 if $\mathrm{a}=\mathrm{b}$, and 0 if $\mathrm{a} \neq \mathrm{b}$. That is, the two subscripts should agree for the Kronecker delta to have value 1 . Otherwise, its value is 0 .

We refer to the neurons with two subscripts, one for the node it refers to, and the other for the order of that node in the path. Therefore, an element of the weight matrix for a connection between two neurons needs to have four subscripts, with a comma after two 
of the subscripts. An example is $W_{x i, y j}$ referring to the weight on the connection between the $(x i)$ neuron and the $(y j)$ neuron.

Now, rewriting (3.3), (3.5), and (3.2) in such a way as to take into account the representation of the neurons with double indices, we get

$$
\begin{aligned}
& \frac{d U_{x i}}{d t}=-\frac{U_{x i}}{\tau}+\sum_{\substack { y=1 \\
\begin{subarray}{c}{j=1 \\
j \neq y{ y = 1 \\
\begin{subarray} { c } { j = 1 \\
j \neq y } }\end{subarray}}^{n} W_{x i, y j} V_{y j}+I_{x i} \\
& \frac{d U_{x i}}{d t}=-\frac{U_{x i}}{\tau}-\frac{\partial E}{\partial V_{x i}} \\
& V_{x i}=\frac{1}{1+e^{-\lambda_{x i} U_{x i}}}
\end{aligned}
$$

By substituting (4.5) in (4.6b), the dynamic equations of the neural network is obtained:

$$
\begin{aligned}
\frac{d U_{x i}}{d t}= & -\frac{U_{x i}}{\tau}-\frac{T_{1}}{2} C_{x i}\left(1-\delta_{x d} \cdot \delta_{i s}\right)-\frac{T_{2}}{2} P_{x i}\left(1-\delta_{x d} \cdot \delta_{i s}\right) \\
& -T_{3} \sum_{\substack{y=1 \\
y \neq x}}^{n}\left(V_{x y}-V_{y x}\right)+T_{3} \sum_{\substack{y=1 \\
y \neq i}}^{n}\left(V_{i y}-V_{y i}\right) \\
& -\frac{T_{4}}{2}\left(1-2 V_{x i}\right)+\frac{T_{5}}{2} \delta_{x d} \cdot \delta_{i s}
\end{aligned}
$$

where $\delta$ is the Kronecker delta defined by

$$
\begin{aligned}
\delta_{a b} & =1 & & \text { if } \mathrm{a}=\mathrm{b} \\
& =0 & & \text { otherwise }
\end{aligned}
$$

By comparing the corresponding coefficients in (4.6a) and (4.8), the biases and the connection weights are derived: 


$$
\begin{aligned}
& I_{x i}=-\frac{T_{1}}{2} C_{x i}\left(1-\delta_{x d} \cdot \delta_{i s}\right)-\frac{T_{2}}{2} P_{x i}\left(1-\delta_{x d} \cdot \delta_{i s}\right)-\frac{T_{4}}{2}+\frac{T_{5}}{2} \delta_{x d} \delta_{i s} \\
& =\left\{\frac{T_{5}}{2}-\frac{T_{4}}{2} \quad \text { if }(x, i)=(d, s)\right. \\
& =\left\{\begin{array}{l}
2 \\
-\frac{T_{1}}{2} C_{x i}-\frac{T_{2}}{2} P_{x i}-\frac{T_{4}}{2} \quad \text { otherwise }
\end{array}\right. \\
& W_{r i, y j}=T_{4} \delta_{x y} \delta_{i j}-T_{3} \delta_{x y}-T_{3} \delta_{j i}+T_{3} \delta_{j x}+T_{3} \delta_{i y}
\end{aligned}
$$

The first term in (4.10) represents excitatory self-feedbacks, and the second and third terms represent local inhibitory connections among neurons in the same row and in the same column, respectively. The last two terms represent excitatory cross-connections among neurons.

An advantage of the proposed model is that it maps the data (here defined by link costs and node connectivity information) into the biases rather than into the neural interconnections. This is due to the fact that the data terms are associated with linear rather than quadratic expressions in the energy function (4.5). One advantage of the proposed representation scheme is the flexibility reflected by the fact that the link costs $C_{x i}$ 's and the network topology information, embedded in the $P_{x i}$ 's terms, can be changed through the biases. Since, in this case, we do not have to modify the internal parameters of the neural network architecture, then the proposed algorithm can adapt rapidly to changes in network topology and link costs. This will make the neural network algorithm very attractive to operate in real time. Another advantage is that the interconnections weights do not depend on a particular source or destination. Hence the neural network can find the shortest path between any given two nodes by properly choosing the input biases as given in (4.9). 


\subsection{Simulation Results}

\subsubsection{Choosing the Values of $T_{1}, T_{2}, T_{3}, T_{4}, T_{5}$ and Neural Transfer}

\section{Parameter $\lambda$}

There is not very much guidance in the literature as to how to choose the parameter sets in general for using a Hopfield neural network to solve an optimization problem like routing problem. In our simulations, an appropriate general size of the parameters is found using a trail and error approach. The results refer to parameter sets at or near $T_{1}=950 ; T_{2}=2500 ; T_{3}=1500 ; T_{4}=475 ; T_{5}=2500$. Experimentally we have found that there is a compromise between choosing a small or a large value for the neural transfer parameter $\lambda$. While a large $\lambda$ gives rise to a fast neural response for which the solution is not always a global minimum, a small $\lambda$ yields a slower response which can guarantee an optimum solution. In fact, it is observed that the ability of the routing neural algorithm to separate between an optimum and a very good solution is also enforced by the smoothness of the neural transfer function (which corresponds to a low neural transfer function $\lambda$ ). This is explained by the fact that a large $\lambda$ (such as 100) may not allow enough time for a neuron to optimize its performance, as the neural response time becomes very fast and hence less accurate. Therefore, in order to allow the neurons' dynamics to wander freely in their state space, in the search for the global minima, I have chosen $\lambda=5$.

\subsubsection{Inputs}

The inputs to the network are chosen arbitrarily. A problem may arise that the network will get stuck at a solution corresponding to local minimum. To avoid such an occurrence, random noise can be added. Usually the input at each neuron is taken as a constant times the number of nodes and this is adjusted by adding a random number, which may be different for different neurons. 


\subsubsection{Activations, Outputs, and Their Updating}

I denote the activation of the neuron in the $i^{\text {th }}$ row and $j^{\text {th }}$ column by $u_{i j}$, and the output is denoted by $V_{i j}$. A time constant $\tau$, and a gain $\lambda$ are used as well.

The change in the activation is then given by $\Delta u_{i j}$, where:

$$
\begin{aligned}
\Delta u_{i j}=\Delta t\left(\operatorname{Term}_{1}+\operatorname{Term}_{2}+\operatorname{Term}_{3}+\operatorname{Term}_{4}+\operatorname{Term}_{5}+\operatorname{Term}_{6}\right) \\
\operatorname{Term}_{1}=\frac{-u_{i j}}{\tau} \\
\operatorname{Term}_{2}=-\frac{T_{1}}{2} C_{x i}\left(1-\delta_{x d} \cdot \delta_{i s}\right) \\
\operatorname{Term}_{3}=-\frac{T_{2}}{2} P_{x i}\left(1-\delta_{x d} \cdot \delta_{i s}\right) \\
\operatorname{Term}_{4}=-T_{3} \sum_{\substack{y=1 \\
y \neq x}}^{n}\left(V_{x y}-V_{y x}\right)+T_{3} \sum_{\substack{y=1 \\
y \neq i}}^{n}\left(V_{i y}-V_{y i}\right) \\
\operatorname{Term}_{5}=-\frac{T_{4}}{2}\left(1-2 V_{x i}\right) \\
\operatorname{Term}_{6}=\frac{T_{5}}{2} \delta_{x d} \cdot \delta_{i s}
\end{aligned}
$$

To update the activation of the neuron in the $i^{\text {th }}$ row and $j^{\text {th }}$ column, I take:

$$
u_{i j n e w}=u_{i j o l d}+\Delta u_{i j}
$$

The output of a neuron in the $i^{\text {th }}$ row and $j^{\text {th }}$ column is calculated from:

$$
V_{i j}=\left(1+\tanh \left(\lambda u_{i j}\right)\right) / 2
$$


The function used here is the hyperbolic tangent function. The output of each neuron is calculated after updating the activation. Ideally, we want to get the outputs as 0's and 1's, to represent a path that satisfies the conditions of the problem. But the hyperbolic tangent function gives a real number, and we have to settle for a close enough value to 1 or 0 . We may get, for example, 0.97 instead of 1 , or 0.06 instead of 0 . The solution is to be obtained from such values by rounding up or down so that 1 or 0 will be used, as the case may be.

Accordingly, the simulation consisted of observing and updating the neuron outputs at incremental time steps $\Delta t$.In addition, the time constant $\tau$ of each neuron is set to 1 without any loss of generality. Simulations have shown that a good value for $\Delta t$ is $10^{-5}$. Reducing this tolerably small value increases the simulation time without improving the results. The simulation program is written in $\mathrm{C}++$. For demonstration, some combination of networks will be considered next [For complete output listing see Appendix A].

\section{Example 1}

The five-node network shown in Figure 4.2(a) is considered. There are six feasible paths between the source node 1 and the destination node 5, shown in Figure 4.2(b). The simulation is conducted with two goals in mind: first to test the performance of the proposed neural network algorithm and second to investigate the impact of various parameters on the quality of the solution. The final output of the neural network is shown in Figure 4.2(c). 


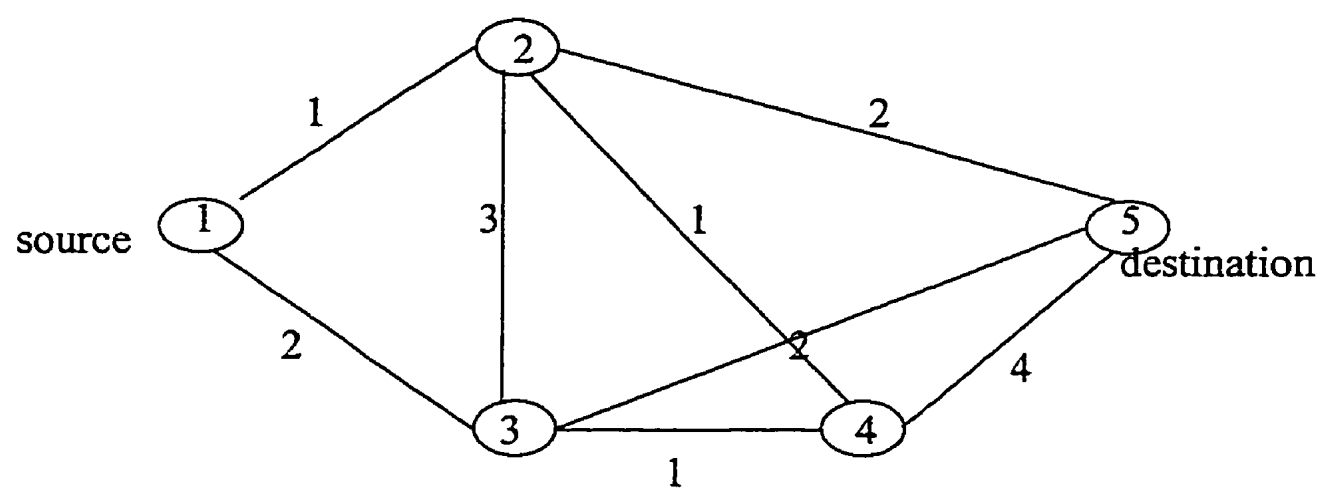

Figure 4.2(a) Network showing link costs

(b) The feasible paths are:

Path 1: 1-2-5 with total cost of 3

Path 2: 1-2-4-5 with total cost of 6

Path 3: 1-2-3-5 with total cost of 6

Path 4: 1-2-3-4-5 with total cost of 9

Path 5: 1-3-5 with total cost of 4

Path 6: 1-3-4-5 with total cost of 7

Table 4.1 (c) The Neural Network outpu

\begin{tabular}{|l|l|l|l|l|l|}
\hline & 1 & 2 & 3 & 4 & 5 \\
\hline 1 & 0 & 1 & 0 & 0 & 0 \\
\hline 2 & 0 & 0 & 0 & 0 & 1 \\
\hline 3 & 0 & 0 & 0 & 0 & 0 \\
\hline 4 & 0 & 0 & 0 & 0 & 0 \\
\hline 5 & 1 & 0 & 0 & 0 & 0 \\
\hline
\end{tabular}




\section{Example 2}

Figure 4.3 shows a network with six nodes and the output of the neural network.

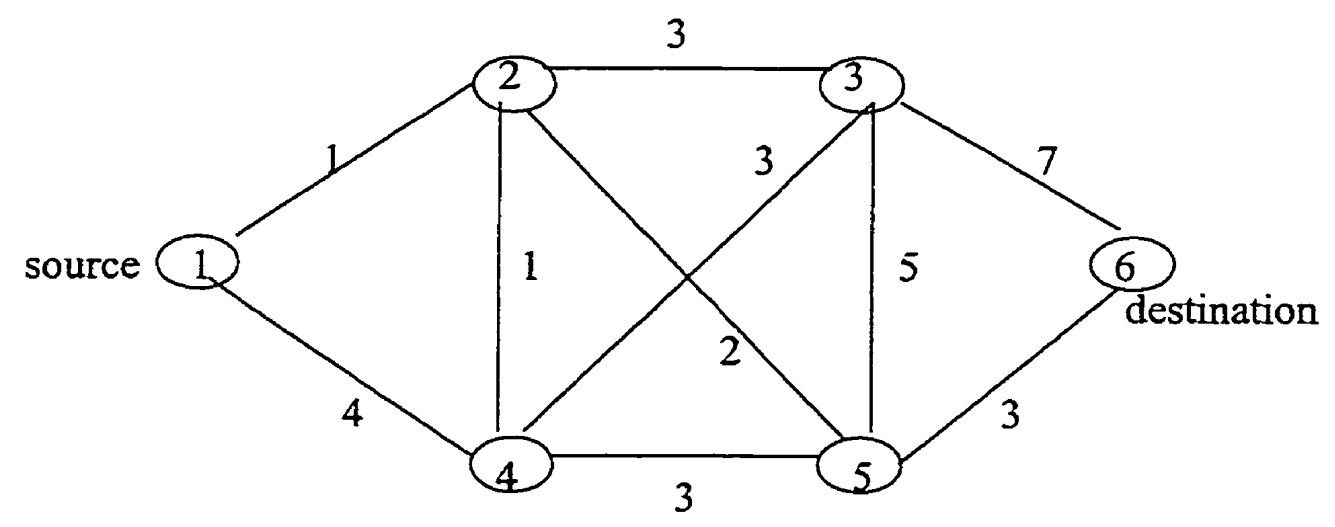

Figure 4.3 A six-node network

Table 4.2 The neural network output

\begin{tabular}{|l|l|l|l|l|l|l|}
\hline & 1 & 2 & 3 & 4 & 5 & 6 \\
\hline 1 & 0 & 1 & 0 & 0 & 0 & 0 \\
\hline 2 & 0 & 0 & 0 & 0 & 1 & 0 \\
\hline 3 & 0 & 0 & 0 & 0 & 0 & 0 \\
\hline 4 & 0 & 0 & 0 & 0 & 0 & 0 \\
\hline 5 & 0 & 0 & 0 & 0 & 0 & 1 \\
\hline 6 & 1 & 0 & 0 & 0 & 0 & 0 \\
\hline
\end{tabular}




\section{Example 3}

In this example a five-node network is considered.

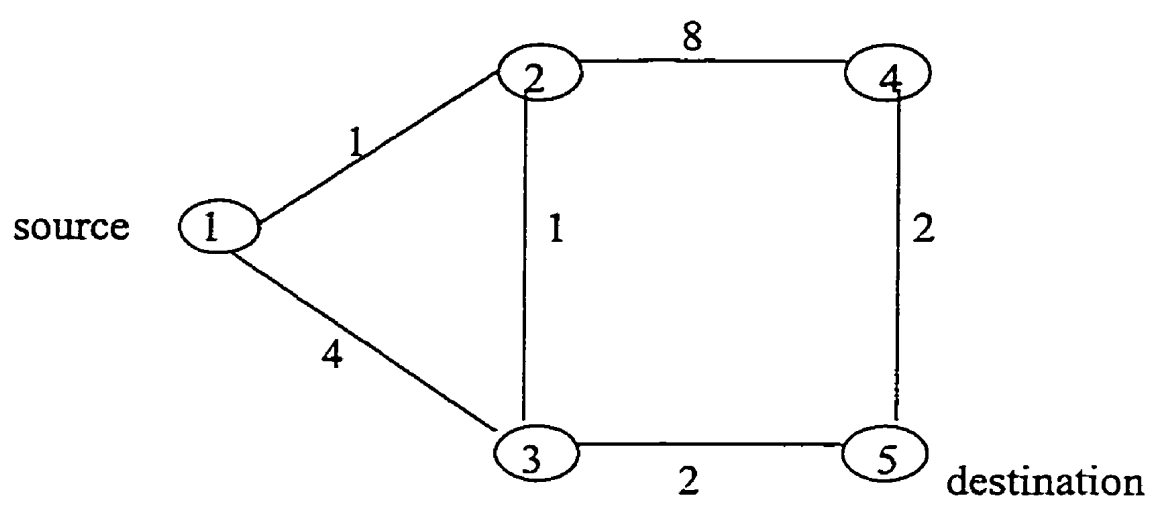

Figure 4.4 A five-node network

Table 4.3 The routing table when source $=1$ and destination

\begin{tabular}{|l|l|l|l|l|l|}
\hline & 1 & 2 & 3 & 4 & 5 \\
\hline 1 & 0 & 1 & 0 & 0 & 0 \\
\hline 2 & 0 & 0 & 1 & 0 & 0 \\
\hline 3 & 0 & 0 & 0 & 0 & 1 \\
\hline 4 & 0 & 0 & 0 & 0 & 0 \\
\hline 5 & 1 & 0 & 0 & 0 & 0 \\
\hline
\end{tabular}




\section{Example 4}

In this example we show the performance of our model on the ten-node network [Figure 4.5] used by Cavalieri Al.[14]. Tables 4.4 and 4.5 show the performance of our model. The result is compared with the data provided in [14]. The performance is better in terms of both number of iterations and optimality of the result. Furthermore our parameters setting has been kept unchanged in spite of changes in the network topology and in the source and destination nodes, while in the previous work a new set-up was necessary for each case. This is a valuable step toward a practical application based on this approach to a real problem.

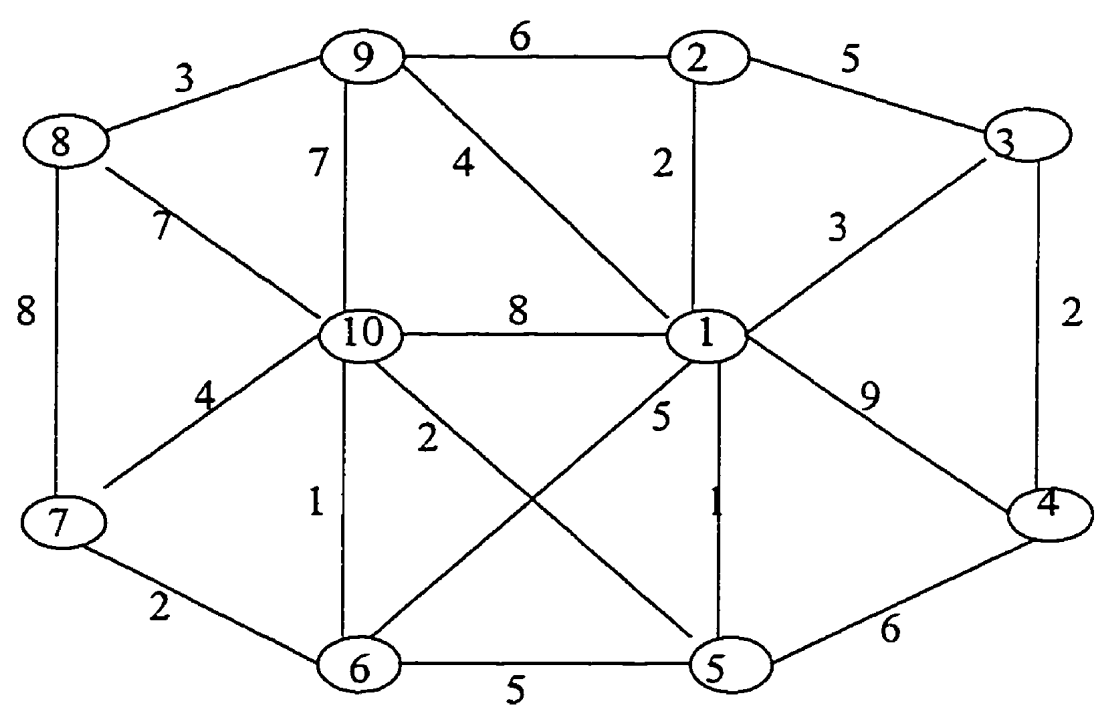

Figure 4.5 A ten-node network 
Table 4.4 Performance of the proposed model vs. Cavalier's. the ten-node networks used in [14] has been used for the test, using node 7 as a source and varying the destination node. The proposed model finds always a valid path.

\begin{tabular}{|l|ccc|ccc|}
\hline \multirow{2}{*}{$\begin{array}{l}\text { Destination } \\
\text { node }\end{array}$} & \multicolumn{3}{|c|}{ Cavalieri's set-up } & \multicolumn{3}{c|}{ Proposed set-up } \\
\hline 1 & optimal & valid & wrong & optimal & valid & wrong \\
\hline 2 & 8 & 0 & 2 & 10 & 0 & 0 \\
\hline 3 & 10 & 0 & 0 & 10 & 0 & 0 \\
\hline 4 & 0 & 10 & 0 & 10 & 0 & 0 \\
\hline 5 & 6 & 4 & 0 & 10 & 0 & 0 \\
\hline 6 & 10 & 0 & 0 & 10 & 0 & 0 \\
\hline 8 & 10 & 0 & 0 & 10 & 0 & 0 \\
\hline 9 & 8 & 1 & 1 & 10 & 0 & 0 \\
\hline 10 & 0 & 9 & 1 & 10 & 0 & 0 \\
\hline
\end{tabular}

Table 4.5 Performance of the proposed model vs. Cavalier's. the ten-node networks used in [14] has been used for the test, using node 10 as a source and varying the destination node. The proposed model finds always a valid path.

\begin{tabular}{|l|ccc|ccc|}
\hline \multirow{2}{*}{$\begin{array}{l}\text { Destination } \\
\text { node }\end{array}$} & \multicolumn{3}{|c|}{ Cavalieri's set-up } & \multicolumn{3}{c|}{ Proposed set-up } \\
\hline 1 & optimal & valid & wron & optimal & valid & wron \\
\hline 2 & 7 & 3 & 0 & 10 & 0 & 0 \\
\hline 3 & 10 & 0 & 0 & 10 & 0 & 0 \\
\hline 4 & 0 & 7 & 3 & 10 & 0 & 0 \\
\hline 5 & 9 & 0 & 1 & 10 & 0 & 0 \\
\hline 6 & 10 & 0 & 0 & 10 & 0 & 0 \\
\hline 7 & 6 & 4 & 0 & 10 & 0 & 0 \\
\hline 8 & 10 & 0 & 0 & 10 & 0 & 0 \\
\hline 9 & 6 & 0 & 4 & 10 & 0 & 0 \\
\hline
\end{tabular}




\section{Example 5}

Fourteen-node connected with twenty four links

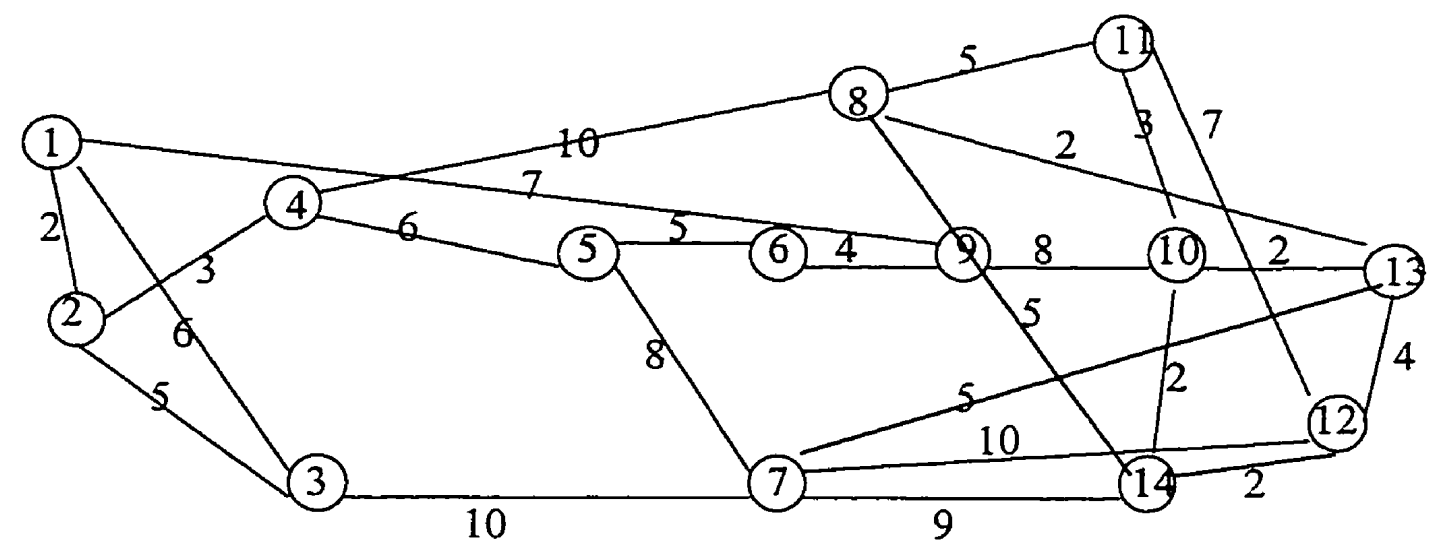

Figure 4.614 nodes and 24 links

Table 4.6 Summary of simulation results for the 14 node network

\begin{tabular}{|l|l|l|l|}
\hline Hops & $\begin{array}{l}\text { Number of optimal } \\
\text { paths }\end{array}$ & $\begin{array}{l}\text { Number of near } \\
\text { optimal paths }\end{array}$ & $\begin{array}{l}\text { Total number of } \\
\text { paths }\end{array}$ \\
\hline 1 & 42 & 0 & 42 \\
\hline 2 & 68 & 2 & 70 \\
\hline 3 & 58 & 12 & 70 \\
\hline
\end{tabular}


Table 4.7 A Comparison of all non-optimal simulation routes with optimal routes

\begin{tabular}{|l|l|l|l|l|l|}
\hline Source & Destination & $\begin{array}{l}\text { Simulation } \\
\text { path }\end{array}$ & Cost & Optimal path & Cost \\
\hline 3 & 5 & $3-7-5$ & 18 & $3-2-4-5$ & 14 \\
\hline 3 & 6 & $3-7-5-6$ & 23 & $3-2-4-5-6$ & 20 \\
\hline 3 & 10 & $3-1-9-10$ & 21 & $3-7-13-10$ & 17 \\
\hline 5 & 3 & $5-7-3$ & 18 & $5-4-2-3$ & 14 \\
\hline 5 & 11 & $5-4-8-11$ & 21 & $5-6-9-10-11$ & 20 \\
\hline 5 & 14 & $5-4-8-14$ & 21 & $5-6-9-10-14$ & 19 \\
\hline 6 & 3 & $6-9-1-3$ & 17 & $6-5-4-2-3$ & 16 \\
\hline 6 & 12 & $6-5-7-12$ & 23 & $6-9-10-14-12$ & 16 \\
\hline 6 & 8 & $6-5-4-8$ & 21 & $6-9-10-14-8$ & 14 \\
\hline 8 & 6 & $8-4-5-6$ & 21 & $8-14-10-9-6$ & 19 \\
\hline 10 & 3 & $10-9-1-3$ & 21 & $10-13-7-3$ & 17 \\
\hline 11 & 5 & $11-8-4-5$ & 21 & $11-10-9-6-5$ & 20 \\
\hline 12 & 6 & $12-7-5-6$ & 24 & $12-14-10-9-6$ & 16 \\
\hline 14 & 5 & $14-8-4-5$ & 21 & $14-10-9-6-5$ & 19 \\
\hline
\end{tabular}

All of the source-destination paths selected were valid and $97.3 \%$ contained the optimum path. The neural network was able to find optimal paths where the path was fairly short. The $\mathbf{2 . 7 \%}$ of nonoptimal paths were mainly longer paths which contained many hops. As the data shows in tables $b \& c$, the neural network had greater difficulty converging to a global minimum when the optimum path took more hops to get from the source to the destination than did another valid path. Usually, the neural network will favor a path which requires a smaller number of hops, even though the optimum path takes more hops. This can be attributed to how the updates are performed on the neural network. The 
updates are done left to right (from source to destination) and have introduced an unwanted bias toward selecting paths that have a smaller number of hops versus the shortest total distance.

\subsection{Comparison Between Neural Network Based Algorithm and Some Conventional Algorithms}

The commonly used routing algorithms are the Bellman-Ford algorithm and Dijkstra's algorithm. The Bellman-Ford algorithm iterates on the number of hops in a path. It is based on first finding optimal paths from a prescribed source node to all other nodes (subject to the constraint that no path contains more than one hop), then finding the optimal path subject to the constraint no path contains more than two hops, and so forth. The amount of computation is at worst $O\left(N^{3}\right)$ [3]. Dijkstra's algorithm iterates on the length of a path. The general idea is to find the shortest paths in order of increasing path length. It first finds the shortest path from some node to the destination node and establishes this path. It then finds the next shortest path and establishes it, and so forth. The number of computations in the worst case is $O\left(N^{2}\right)$ [3]. The Floyd-Warshall algorithm iterates on nodes allowed as intermediate nodes in paths. It computes shortest paths between all pairs of nodes. The Floyd- Warshall algorithm is used in some networks with centralized routing such as TYMNET [3]. The number of computation in the worst case is $O\left(N^{3}\right)[3]$.

In the Hopfield neural network algorithm proposed, each processing element making up the Hopfield network is characterized by $N^{2}$ inputs. The operations it has to perform in each iteration are $N^{2}$ multiplications between the inputs and the weights of each connection, and a sum of the previous multiplication outcomes.. 
The massively parallel architecture and the hypothesis adopted, according to which activation of all the neurons is synchronous, allows as to state that the processing time in each single iteration coincides with the time corresponding to each processing unit, that is:

$$
T_{i}=f\left(N^{2}\right)
$$

In other words, the processing time $T_{i}$ for each single iteration depends on the number of neurons in the Hopfield network. Indicating the number of iterations required for all network to reach full convergence as $K$, the overall time $T$ necessary for a solution to be reached is equal to:

$$
T=K . T_{i}
$$

In a VLSI implementation, where each processing element is realized through operational amplifiers [19], each time value $\mathcal{T}_{i}$ is practically independent of the number of inputs (i.e., $N^{2}$ ).

In addition, in [20] it is shown that the number of iterations needed for a Hopfield network to reach a stable solution in an optimization problem does not depend on its complexity (i.e., the number of neurons in the network).

From these two considerations it can be concluded that the processing times of the solution proposed are practically independent of its complexity, being only linked rather to the operating speed of the analog hardware used, the performance of which depends on the technological evolution of the VLSI components. 
The neural routing algorithm can be considered as an isolated routing algorithm in the sense that no information exchanged between nodes. In distributed routing algorithms, such as the distributed asynchronous Bellman-Ford algorithm, the computation of paths is shared among network nodes with information exchanged between them. Dijkstra's algorithm requires to know the current link status of the network to compute the shortest paths. Hence each node needs to broadcast to all other nodes its connectivity to its neighbors. All the algorithms including the Hopfield neural network are able to adapt to changes in the network. Table 4.8 gives a comparison between the Hopfield algorithm and some conventional algorithms, where $N$ is the number of nodes in the network.

Table 4.8 Comparison of the neural approach and other routing algorithms.

\begin{tabular}{|l|l|l|l|}
\hline Complexity & Computation & Communication & $\begin{array}{l}\text { Memory } \\
\text { Required }\end{array}$ \\
\hline $\begin{array}{l}\text { Bellman-Ford } \\
\text { Dijkstra's }\end{array}$ & $\begin{array}{l}O\left(N^{3}\right) \\
\text { the latest link cost }\end{array}$ & $\begin{array}{l}\text { Each node broadcasts to its neighbors of } \\
\text { about its connectivity to neighbor nodes }\end{array}$ & Yes \\
\hline Hopfield & $\begin{array}{l}\text { Based on the } \\
\text { speed of the } \\
\text { analog hardware } \\
\text { used }\end{array}$ & None & No \\
\hline
\end{tabular}




\title{
Chapter 5
}

\section{Conclusions and Future Research}

\begin{abstract}
A new solution to the routing problem is proposed, using a Hopfield neural network. The architecture of the model and the simulation results are given. Convergence to optimal solutions is guaranteed with an acceptable percentage of error. This is inevitable in approaches of this kind, as has already been pointed out in similar cases [2], and is due to the high number of local minima the energy function presents. One of the most interesting results obtained during the experimental tests is the network's capacity to acquire an awareness of the network topology alone, and its ability to adapt itself to variations in the costs of the links in the network.
\end{abstract}

The fact that a Hopfield network is difficult to simulate [20], makes the model not always able to generate a valid solution especially when applied to large size systems [as can be seen from Figure 5.1]. Solutions, especially when simulating networks of higher dimension, can only be found after testing hundreds of different parameter sets, chosen by experience. The relationship between the parameter sets and the network size is not completely clear yet and needs further investigation.

Since the Hopfield neural network can be implemented by an analog circuit, which is one order faster than any digital table lookup implementation and is smaller in size, it could be applicable to situations where time and space are critical and near optimal solution is acceptable. 


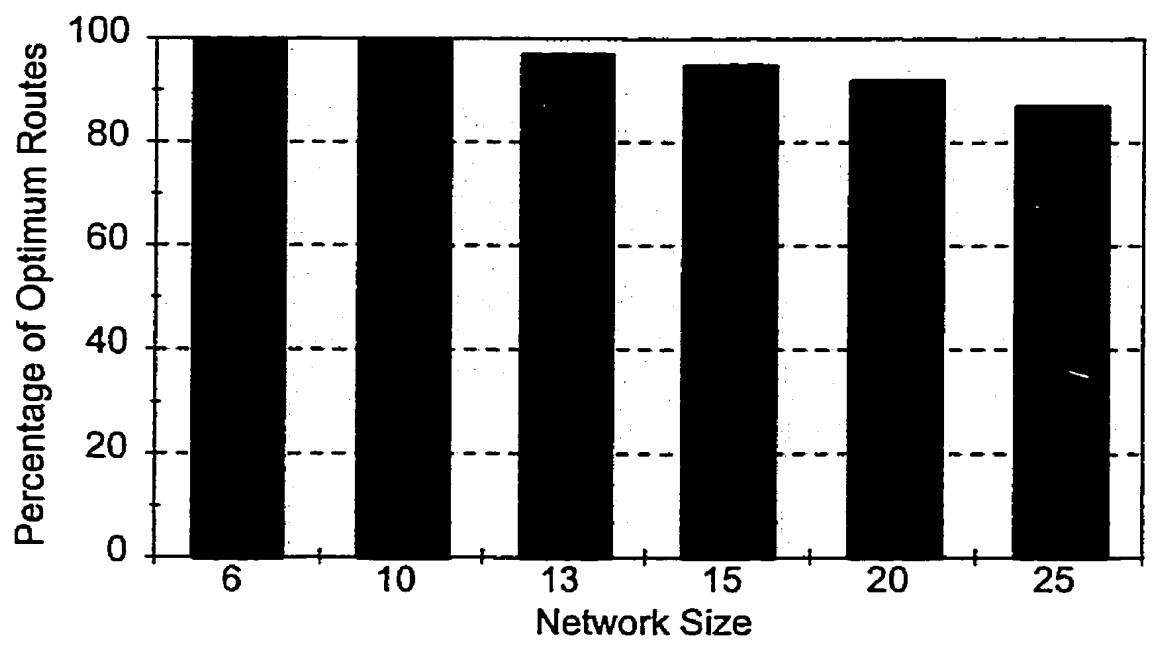

Figure 5.1 Optimal route percentage for different networks (as can be seen from the graph, the number of optimum routes obtained by the model decreases when the network size increases. This is due to the fact that the model is difficult to simulate because of the required tuning of the parameters $T_{1}, T_{2}, T_{3}, T_{4}$, and $T_{5}$.

\subsection{Suggestions for Future Research}

\subsubsection{Tuning the Parameter Sets}

The main drawback of Hopfield neural networks is related mainly to the choice of the value of the parameters used. This makes choosing the right set a very important issue, since it affects the overall output of the network. Tuning these parameter sets using a trial and error approach is time consuming and each time the neural network changed, the parameter set need to be changed too. A general formula showing how these parameter sets can be chosen is an essential instead of trying hundred of parameter sets.

\subsubsection{Alternative Neural Network Structures}

Regarding another neural network structure, the self organising map from T. Kohonen [22] seemed to be more promising than to spend time on tuning parameter sets. 
Neural Networks can generally be subdivided into three main groups [8] :

1. feedback networks including Hopfield and Boltzman networks.

2. feedforward networks including Backpropagation and related structures

3. unsupervised learning networks including self organising feature maps

Especially the self organising feature map of the last network type reflects in its structure spatial and temporal statistical properties of the input vector space, which led us to believe, that these maps can be applied to the routing problem. As pointed out in chapter 2 , in computer networks there arise the problem to adapt the routing algorithms to the topology of the network and to the actual traffic load, which is changing over time. Adaptive routing algorithms require exponentially increasing time in relation to the number of nodes in the network. It must be possible to reduce this required time, if the topology of the network and the cost of the links are mapped to the topology and weight connection of a neural network [Figure 5.2] . Further work needed to evaluate the applicability of this model and its extensions to the routing problem.

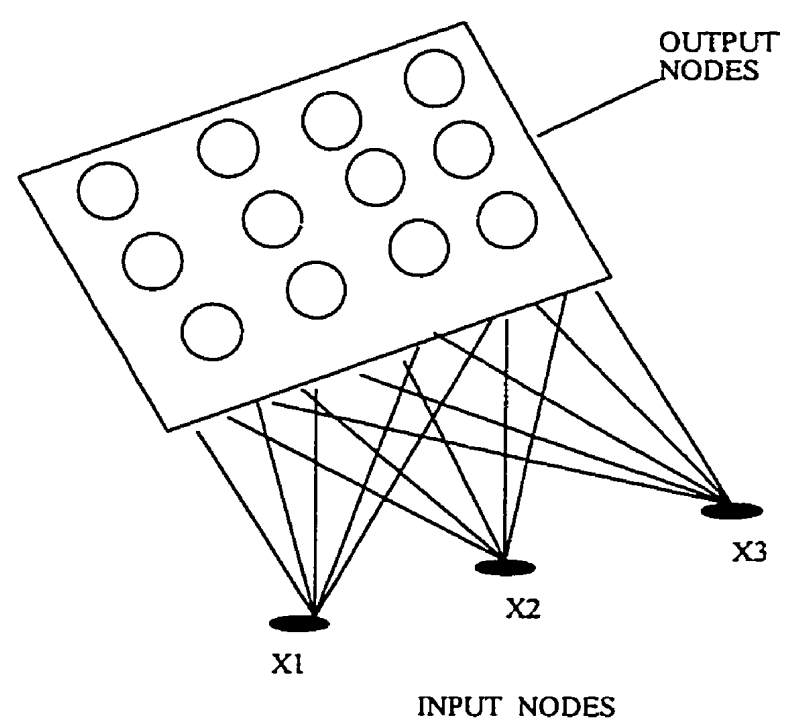

Figure 5.2 Structure of a two-dimensional array of formal neurons, each fully connected with a layer of input neurons $x_{\mathrm{I}} \ldots x_{N}$ 


\section{Chapter 6}

\section{References}

1- Radia Perlman, Interconnections: Bridges and Routers, Second edition, AddisonWesley Professional Computing Series, Reading, Massachusetts, 1992.

2- J. J. Hopfield and D. W. Tank, Neural Computation of decisions in optimization problems, Biological Cybernetics, Vol. 52, PP. 141-152, 1985.

3- Dimitri Bertsekas and Robert Gallager, Data Networks, Second edition, Prentice Hall, New Jersey, 1992.

4- C. N. Zhang, M. Wang and W. K. Chou, A bidirectional vector associative memory architecture with application to neural networks, VLSI Technology, Systems, and Applications, Proceedings of Technical Papers. 1995.

5- I. Cidon, I. S. Gopal, M. Kaplan, and S. Kutten, Distributed Control for PARIS, Proc. Of the $9^{\text {th }}$ Annual ACM Symposium on Principles of Computing, Quebec, Canada, 1990.

6- D. Bertsekas, Dynamic Programming: Deterministic and Stochastic Models, Englewood Cliffs, NJ, Prentice Hall, 1987. 
7- D. Bertsekas, Linear Network Optimization: Algorithms and Codes, MIT Press, Cambridge, MA, 1991.

8- R. P. Lippmann, An introduction to computing with neural nets, IEEE ASSP Magazine, pp. 4-22, April 1987.

9- J. Madrenas, J. M. Moreno, J. Cabestany, J. Seco, and S. Usui, A bit-serial VLSI processor for kernel-based classifiers, Neural Networks for Signal Processing, Proceeding of the 1996 Signal Processing Society Workshop, pp. 549-558, 1996.

10- M. L. Campbell and S. T. Toborg, 3-D wafer scale architectures for neural network computing, IEEE Transactions on Components, Packaging, and Manufacturing Technology, Volume 167, pp. 646-655, 1993.

11- J. J. Hopfield and D. W. Tank, Computing with neural circuits: A model, Science: 233, pp. 625-633, 1986.

12- G. Frisiani, T. Parisini, L. Siccardi, and R. Zoppoli, Team theory and backpropagation for dynamic routing in communication networks, IEEE Transaction on Neural network, pp. 325-332, 1991.

13- H. E. Rauch and T. Winarske, Neural networks for routing communication traffic", IEEE Con. Sys. Mag., pp. 26-30, Apr. 1988.

14- S. Cavalieri A. Di Stefano and O. Mirabella, Optimal path determination in a graph by Hopfield neural network, Neural Networks, Vol. 1, no. 2, pp. 397-404, 1994. 
15- N. Funabiki, S. Nishikawa, and S. Tajima, A binary neural network approach for net assignment problems, 1996 International Conference on Neural Network, Volume 4, pp. 2188-2193, 1996.

16- Takefuji Y., CMOS layout design of the hysteresis McCulloch-Pitts neuron, Electronics Letters, Vol. 26 25, pp. 2093-2095, Dec. 1990.

17- J.J Hopfield, Neurons with Graded Response have collective computation properties like those of two-state neurons, Proceedings National Academic of Science, USA, Vol. 81, PP. 3088-3092, 1984.

18- L. Zhang and S. C. A. Thomopoulos, Neural network implementation of the shortest path algorithm for traffic routing in communication networks, in Proc. Int. Joint Conf. Neural Networks, June 1989.

19- D. J. Amit Modeling Brain Function, Cambridge University Press, pp. 461-480, 1989.

20- S. V. Aiyer, M. Niranjan, and F. Fallside A theoretical investigation into the performance of the Hopfield model, IEEE Transactions on Neural Networks, pp. 204215, January 1990.

21- Mustafa K. Ali and Faouzi Kamoun, Neural Networks for Shortest Path Computation and Routing in Computer Networks, IEEE Transactions on Neural Networks, Vol. 4, NO. 6, pp. 941-953, November 1993.

22- T. Kohonen, The Self-Organizing Map, Proceeding of the IEEE, Vol. 78, No.9, September 1990. 


\section{Appendix A:}

\section{Complete Output of a five-node network and a six-node network}

Output of a network with five nodes where source is 1 and destination is 5

// The cost of the links are solicited.

// For nonexisting links this cost is set to be zero

Cost Matrix

0233415

206678

360090

$4 \begin{array}{lllll}4 & 9 & 0 & 11\end{array}$

1580110

// $25 \times 25$ matrix of weights. There are 25 neurons in the network

Weight Matrix

$\begin{array}{llllll}475 & 0 & 0 & 0 & 0\end{array}$

$0 \begin{array}{lllll}0 & 0 & 0 & 0 & 0\end{array}$

$0 \begin{array}{lllll}0 & 0 & 0 & 0 & 0\end{array}$

$\begin{array}{lllll}0 & 0 & 0 & 0 & 0\end{array}$

$\begin{array}{lllll}0 & 0 & 0 & 0 & 0\end{array}$

$\begin{array}{llllll}0 & 475 & -1500 & -1500 & -1500 \\ 3000 & 0 & 1500 & 1500 & 1500 \\ 1500 & -1500 & 0 & 0 & 0 & \\ 1500 & -1500 & 0 & 0 & 0 & \\ 1500 & -1500 & 0 & 0 & 0 & \end{array}$




$$
\begin{array}{llllll}
0 & -1500 & 475 & -1500 & -1500 \\
1500 & 0 & -1500 & 0 & 0 & \\
3000 & 1500 & 0 & 1500 & 1500 \\
1500 & 0 & -1500 & 0 & 0 & \\
1500 & 0 & -1500 & 0 & 0 &
\end{array}
$$

$$
\begin{array}{llllll}
0 & -1500 & -1500 & 475 & -1500 \\
1500 & 0 & 0 & -1500 & 0 & \\
1500 & 0 & 0 & -1500 & 0 & \\
3000 & 1500 & 1500 & 0 & 1500 \\
1500 & 0 & 0 & -1500 & 0 &
\end{array}
$$

$$
\begin{array}{llllll}
0 & -1500 & -1500 & -1500 & 475 \\
1500 & 0 & 0 & 0 & -1500 & \\
1500 & 0 & 0 & 0 & -1500 & \\
1500 & 0 & 0 & 0 & -1500 & \\
3000 & 1500 & 1500 & 1500 & 0
\end{array}
$$

$$
\begin{aligned}
& \begin{array}{lllll}
0 & 3000 & 1500 & 1500 & 1500
\end{array} \\
& \begin{array}{llllll}
475 & 0 & -1500 & -1500 & -1500
\end{array} \\
& \begin{array}{lllll}
-1500 & 1500 & 0 & 0 & 0
\end{array} \\
& \begin{array}{lllll}
-1500 & 1500 & 0 & 0 & 0
\end{array} \\
& \begin{array}{lllll}
-1500 & 1500 & 0 & 0 & 0
\end{array}
\end{aligned}
$$

$\begin{array}{llllll}0 & 0 & 0 & 0 & 0 \\ 0 & 475 & 0 & 0 & 0 \\ 0 & 0 & 0 & 0 & 0 \\ 0 & 0 & 0 & 0 & 0 \\ 0 & 0 & 0 & 0 & 0\end{array}$

$$
\begin{array}{llllll}
0 & 1500 & -1500 & 0 & 0 & \\
-1500 & 0 & 475 & -1500 & -1500 \\
1500 & 3000 & 0 & 1500 & 1500 \\
0 & 1500 & -1500 & 0 & 0 & \\
0 & 1500 & -1500 & 0 & 0 &
\end{array}
$$




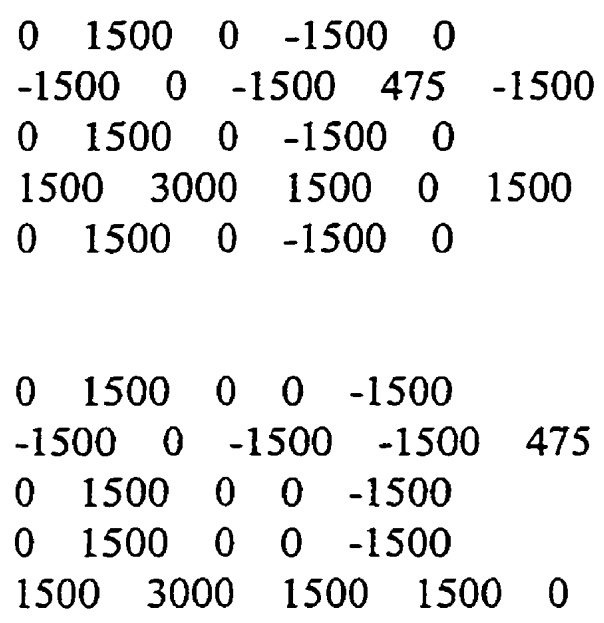

$\begin{array}{lllll}0 & 1500 & 3000 & 1500 & 1500\end{array}$

$\begin{array}{lllll}-1500 & 0 & 1500 & 0 & 0\end{array}$

$\begin{array}{llllll}475 & -1500 & 0 & -1500 & -1500\end{array}$

$\begin{array}{lllll}-1500 & 0 & 1500 & 0 & 0\end{array}$

$\begin{array}{lllll}-1500 & 0 & 1500 & 0 & 0\end{array}$

$\begin{array}{lllll}0 & -1500 & 1500 & 0 & 0\end{array}$

$\begin{array}{lllll}1500 & 0 & 3000 & 1500 & 1500\end{array}$

$\begin{array}{lllll}-1500 & 475 & 0 & -1500 & -1500\end{array}$

$\begin{array}{lllll}0 & -1500 & 1500 & 0 & 0\end{array}$

$\begin{array}{lllll}0 & -1500 & 1500 & 0 & 0\end{array}$

$\begin{array}{llllll}0 & 0 & 0 & 0 & 0 \\ 0 & 0 & 0 & 0 & 0 \\ 0 & 0 & 475 & 0 & 0 \\ 0 & 0 & 0 & 0 & 0 \\ 0 & 0 & 0 & 0 & 0\end{array}$

$\begin{array}{lllll}0 & 0 & 1500 & -1500 & 0\end{array}$

$\begin{array}{llllll}0 & 0 & 1500 & -1500 & 0\end{array}$

$\begin{array}{llllll}-1500 & -1500 & 0 & 475 & -1500\end{array}$

$\begin{array}{lllll}1500 & 1500 & 3000 & 0 & 1500\end{array}$

$\begin{array}{llllll}0 & 0 & 1500 & -1500 & 0\end{array}$ 


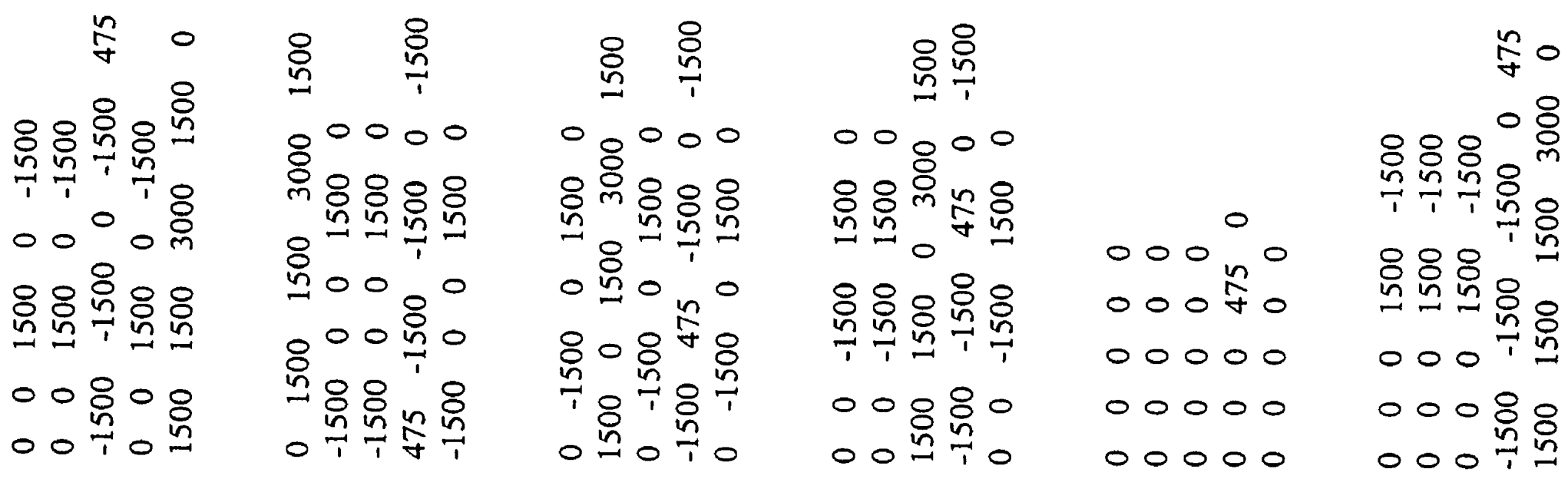




$$
\begin{array}{llllll}
0 & 1500 & 1500 & 1500 & 3000 \\
-1500 & 0 & 0 & 0 & 1500 & \\
-1500 & 0 & 0 & 0 & 1500 & \\
-1500 & 0 & 0 & 0 & 1500 & \\
475 & -1500 & -1500 & -1500 & 0
\end{array}
$$

$\begin{array}{lllll}0 & -1500 & 0 & 0 & 1500\end{array}$

$\begin{array}{lllll}1500 & 0 & 1500 & 1500 & 3000\end{array}$

$\begin{array}{lllll}0 & -1500 & 0 & 0 & 1500\end{array}$

$\begin{array}{lllll}0 & -1500 & 0 & 0 & 1500\end{array}$

$\begin{array}{lllll}-1500 & 475 & -1500 & -1500 & 0\end{array}$

$$
\begin{array}{llllll}
0 & 0 & -1500 & 0 & 1500 & \\
0 & 0 & -1500 & 0 & 1500 & \\
1500 & 1500 & 0 & 1500 & 3000 \\
0 & 0 & -1500 & 0 & 1500 & \\
-1500 & -1500 & 475 & -1500 & 0
\end{array}
$$

$$
\begin{array}{lllllll}
0 & 0 & 0 & -1500 & 1500 & \\
0 & 0 & 0 & -1500 & 1500 & \\
0 & 0 & 0 & -1500 & 1500 & \\
1500 & 1500 & 1500 & 0 & 3000 \\
-1500 & -1500 & -1500 & 475 & 0
\end{array}
$$

$$
\begin{array}{lllll}
0 & 0 & 0 & 0 & 0 \\
0 & 0 & 0 & 0 & 0 \\
0 & 0 & 0 & 0 & 0 \\
0 & 0 & 0 & 0 & 0 \\
0 & 0 & 0 & 0 & 475
\end{array}
$$


initial activations

// initial activation chosen by the system

the activations:

$-102.029991-176.315018-144.989990-132.744995-188.135010$

$-173.809982-104.095016-114.599991-154.279999-272.250000$

$-176.970016-267.189972-343.529968-245.914948-346.614960$

$-291.005035-295.075043-284.899994-389.670044-274.659973$

$-305.884979-333.904999-294.210052-274.724945-347.600037$

// initial output

the outputs

0.2271700 .1075720 .1493280 .1689710 .094694

0.1104930 .2228490 .2017810 .1357130 .036721

0.1068200 .0389300 .0159480 .0496900 .015377

0.0295390 .0281710 .0317130 .0092300 .035711

0.0248290 .0178650 .0284560 .0356850 .015199

1000 iterations completed

// the final activation after 1000 iterations

the activations:

$31.260902-38.566109-60.774864-91.156570-149.450394$

$-130.411118-302.902802-504.180939-714.614258-953.097656$

$-52.630695-219.847778-374.374329-462.944427-624.582336$

$-56.621063-148.819778-233.105103-358.883759-402.549164$

$-253.779373-547.079773-813.591431-1087.381470-1395.025024$

// the final output

the outputs

0.0926980 .8863230 .3253490 .2508890 .142656

0.1729400 .0257100 .0023520 .0001890 .900011

0.3471550 .0667220 .0110690 .0038520 .000556

0.3363830 .1435840 .0574720 .0133000 .007919

0.9454190 .0014070 .0000580 .0000020 .000000 
the route :

1

2

5

cost of route is : 10.000000

Output of a network with six nodes where source is 1 and destination is 6

// The cost of the links are solicited

// For nonexisting links this cost is set to be zero

Cost Matrix

0234460

$\begin{array}{llllll}2 & 0 & 7 & 8 & 9 & 0\end{array}$

$\begin{array}{lllllll}3 & 7 & 0 & 11 & 12 & 9\end{array}$

$\begin{array}{lllllll}4 & 8 & 11 & 0 & 8 & 7\end{array}$

69128006

0009760

// $36 \times 36$ matrix of weights. There are 36 neurons in the network

Weight Matrix

$\begin{array}{llllll}475 & 0 & 0 & 0 & 0 & 0\end{array}$

$\begin{array}{llllll}0 & 0 & 0 & 0 & 0 & 0\end{array}$

$\begin{array}{llllll}0 & 0 & 0 & 0 & 0 & 0\end{array}$

$\begin{array}{llllll}0 & 0 & 0 & 0 & 0 & 0\end{array}$

$\begin{array}{llllll}0 & 0 & 0 & 0 & 0 & 0\end{array}$

$\begin{array}{llllll}0 & 0 & 0 & 0 & 0 & 0\end{array}$

$\begin{array}{llllll}0 & 475 & -1500 & -1500 & -1500 & -1500\end{array}$

$\begin{array}{lllllll}3000 & 0 & 1500 & 1500 & 1500 & 1500\end{array}$

$\begin{array}{llllll}1500 & -1500 & 0 & 0 & 0 & 0\end{array}$

$1500-1500 \quad 0 \quad 00000$

$\begin{array}{llllll}1500 & -1500 & 0 & 0 & 0 & 0\end{array}$ 


$$
\begin{aligned}
& \begin{array}{cccccc}
1500 & -1500 & 0 & 0 & 0 & 0
\end{array} \\
& \begin{array}{llllll}
0 & -1500 & 475 & -1500 & -1500 & -1500
\end{array} \\
& \begin{array}{llllll}
1500 & 0 & -1500 & 0 & 0 & 0
\end{array} \\
& \begin{array}{llllll}
3000 & 1500 & 0 & 1500 & 1500 & 1500
\end{array} \\
& \begin{array}{llllll}
1500 & 0 & -1500 & 0 & 0 & 0
\end{array} \\
& 1500 \quad 0 \quad-1500 \quad 0000
\end{aligned}
$$

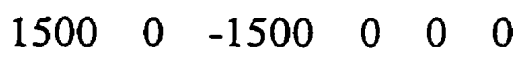

$$
\begin{aligned}
& \begin{array}{llllll}
0 & -1500 & -1500 & 475 & -1500 & -1500
\end{array} \\
& \begin{array}{lllllll}
1500 & 0 & 0 & -1500 & 0 & 0
\end{array} \\
& \begin{array}{lllllll}
1500 & 0 & 0 & -1500 & 0 & 0
\end{array} \\
& \begin{array}{llllll}
3000 & 1500 & 1500 & 0 & 1500 & 1500
\end{array}
\end{aligned}
$$

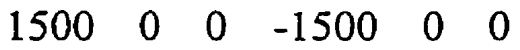

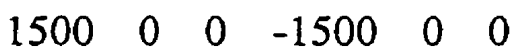

$$
\begin{aligned}
& \begin{array}{llllll}
0 & -1500 & -1500 & -1500 & 475 & -1500
\end{array} \\
& 1500 \quad 000000000
\end{aligned}
$$

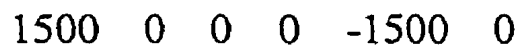

$$
\begin{aligned}
& 1500 \quad 0 \quad 0000-1500 \quad 0 \\
& 3000 \quad 1500 \quad 1500 \quad 1500 \quad 0 \quad 1500 \\
& \begin{array}{lllllll}
1500 & 0 & 0 & 0 & -1500 & 0
\end{array} \\
& \begin{array}{llllll}
0 & -1500 & -1500 & -1500 & -1500 & 475
\end{array} \\
& 1500 \quad 000000000
\end{aligned}
$$

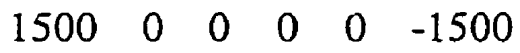

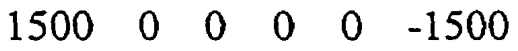

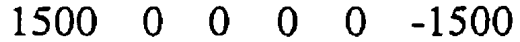

$$
\begin{aligned}
& \begin{array}{llllll}
3000 & 1500 & 1500 & 1500 & 1500 & 0
\end{array} \\
& \begin{array}{llllll}
0 & 3000 & 1500 & 1500 & 1500 & 1500
\end{array} \\
& \begin{array}{llllll}
475 & 0 & -1500 & -1500 & -1500 & -1500
\end{array} \\
& \begin{array}{llllll}
-1500 & 1500 & 0 & 0 & 0 & 0
\end{array} \\
& \begin{array}{llllll}
-1500 & 1500 & 0 & 0 & 0 & 0
\end{array} \\
& \begin{array}{llllll}
-1500 & 1500 & 0 & 0 & 0 & 0
\end{array} \\
& \begin{array}{llllll}
-1500 & 1500 & 0 & 0 & 0 & 0
\end{array}
\end{aligned}
$$




$$
\begin{array}{llllllll}
0 & 0 & 0 & 0 & 0 & 0 \\
0 & 475 & 0 & 0 & 0 & 0 \\
0 & 0 & 0 & 0 & 0 & 0 & & \\
0 & 0 & 0 & 0 & 0 & 0 & & \\
0 & 0 & 0 & 0 & 0 & 0 & & \\
0 & 0 & 0 & 0 & 0 & 0 \\
0 \\
0
\end{array}
$$


인

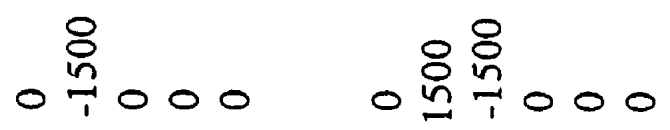

0000000000

00

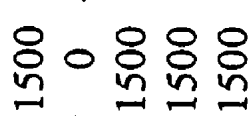

0

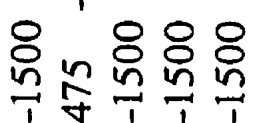

$\circ \stackrel{8}{0}{ }_{1}^{\circ} 000$ 움윰윰유

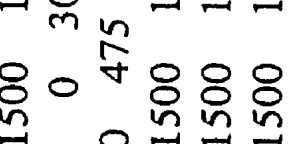

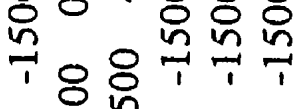
0
운.

00000 00.000 $00{ }^{n} 000$ 00 f 000 000000 000000
号

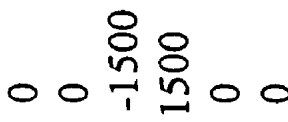
00 no00 윰융융유 ำ 은음운은응

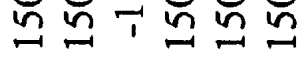

008800 $00 ? 500$ $00^{n} 0_{0}^{n} 0$

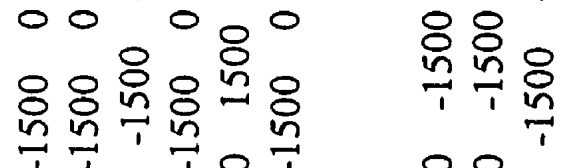
10181 $00^{\circ} 0$

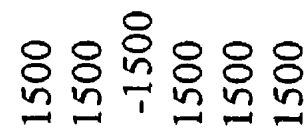
008080 $00 \frac{2}{1} 00$ $\stackrel{n}{*}$

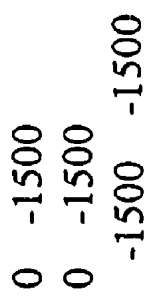
$00^{\circ}$ 88 은 는 008 


$$
\begin{aligned}
& \begin{array}{llllll}
0 & 0 & 1500 & 0 & 0 & -1500
\end{array} \\
& \begin{array}{llllll}
0 & 0 & 1500 & 0 & 0 & -1500
\end{array} \\
& \begin{array}{llllll}
1500 & 1500 & 3000 & 1500 & 1500 & 0
\end{array} \\
& \begin{array}{llllll}
0 & 1500 & 1500 & 3000 & 1500 & 1500
\end{array} \\
& \begin{array}{llllll}
-1500 & 0 & 0 & 1500 & 0 & 0
\end{array} \\
& \begin{array}{llllll}
-1500 & 0 & 0 & 1500 & 0 & 0
\end{array} \\
& \begin{array}{llllll}
475 & -1500 & -1500 & 0 & -1500 & -1500
\end{array} \\
& \begin{array}{llllll}
-1500 & 0 & 0 & 1500 & 0 & 0
\end{array} \\
& \begin{array}{llllll}
-1500 & 0 & 0 & 1500 & 0 & 0
\end{array} \\
& \begin{array}{llllll}
0 & -1500 & 0 & 1500 & 0 & 0
\end{array} \\
& \begin{array}{llllll}
1500 & 0 & 1500 & 3000 & 1500 & 1500
\end{array} \\
& \begin{array}{llllll}
0 & -1500 & 0 & 1500 & 0 & 0
\end{array} \\
& \begin{array}{llllll}
-1500 & 475 & -1500 & 0 & -1500 & -1500
\end{array} \\
& \begin{array}{llllll}
0 & -1500 & 0 & 1500 & 0 & 0
\end{array} \\
& \begin{array}{llllll}
0 & -1500 & 0 & 1500 & 0 & 0
\end{array} \\
& \begin{array}{llllll}
0 & 0 & -1500 & 1500 & 0 & 0
\end{array} \\
& \begin{array}{llllll}
0 & 0 & -1500 & 1500 & 0 & 0
\end{array} \\
& \begin{array}{llllll}
1500 & 1500 & 0 & 3000 & 1500 & 1500
\end{array} \\
& \begin{array}{llllll}
-1500 & -1500 & 475 & 0 & -1500 & -1500
\end{array} \\
& \begin{array}{llllll}
0 & 0 & -1500 & 1500 & 0 & 0
\end{array} \\
& \begin{array}{lllllll}
0 & 0 & -1500 & 1500 & 0 & 0
\end{array} \\
& \begin{array}{lllllll}
0 & 0 & 0 & 1500 & -1500 & 0
\end{array} \\
& \begin{array}{lllllll}
0 & 0 & 0 & 1500 & -1500 & 0
\end{array} \\
& \begin{array}{lllllll}
0 & 0 & 0 & 1500 & -1500 & 0
\end{array} \\
& \begin{array}{llllll}
-1500 & -1500 & -1500 & 0 & 475 & -1500
\end{array} \\
& \begin{array}{llllll}
1500 & 1500 & 1500 & 3000 & 0 & 1500
\end{array}
\end{aligned}
$$




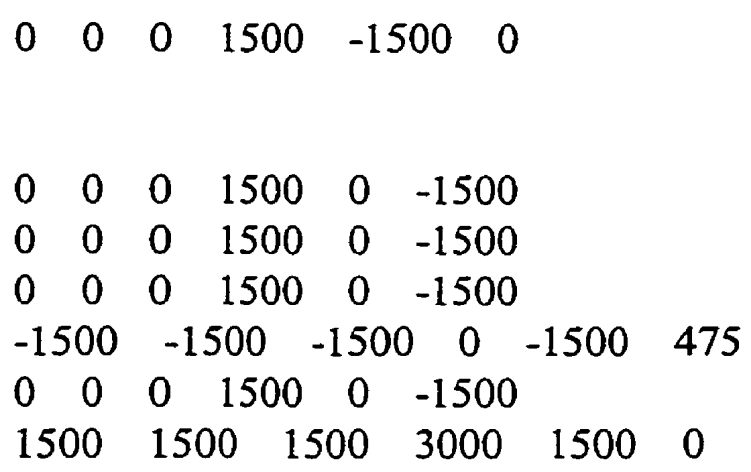

$\begin{array}{llllll}0 & 1500 & 1500 & 1500 & 3000 & 1500\end{array}$

$\begin{array}{lllllll}-1500 & 0 & 0 & 0 & 1500 & 0\end{array}$

$-1500 \quad 0 \quad 00001500 \quad 0$

$\begin{array}{llllll}-1500 & 0 & 0 & 0 & 1500 & 0\end{array}$

$\begin{array}{llllll}475 & -1500 & -1500 & -1500 & 0 & -1500\end{array}$

$\begin{array}{llllll}-1500 & 0 & 0 & 0 & 1500 & 0\end{array}$

$$
\begin{array}{lllllll}
0 & -1500 & 0 & 0 & 1500 & 0 & \\
1500 & 0 & 1500 & 1500 & 3000 & 1500 \\
0 & -1500 & 0 & 0 & 1500 & 0 & \\
0 & -1500 & 0 & 0 & 1500 & 0 & \\
-1500 & 475 & -1500 & -1500 & 0 & -1500 \\
0 & -1500 & 0 & 0 & 1500 & 0
\end{array}
$$

$$
\begin{array}{lllllll}
0 & 0 & -1500 & 0 & 1500 & 0 & \\
0 & 0 & -1500 & 0 & 1500 & 0 & \\
1500 & 1500 & 0 & 1500 & 3000 & 1500 \\
0 & 0 & -1500 & 0 & 1500 & 0 & \\
-1500 & -1500 & 475 & -1500 & 0 & -1500 \\
0 & 0 & -1500 & 0 & 1500 & 0 &
\end{array}
$$

$$
\begin{array}{lllllll}
0 & 0 & 0 & -1500 & 1500 & 0 & \\
0 & 0 & 0 & -1500 & 1500 & 0 & \\
0 & 0 & 0 & -1500 & 1500 & 0 & \\
1500 & 1500 & 1500 & 0 & 3000 & 1500 \\
-1500 & -1500 & -1500 & 475 & 0 & -1500 \\
0 & 0 & 0 & -1500 & 1500 & 0 &
\end{array}
$$


$\begin{array}{llllll}0 & 0 & 0 & 0 & 0 & 0\end{array}$

$\begin{array}{llllll}0 & 0 & 0 & 0 & 0 & 0\end{array}$

$\begin{array}{llllll}0 & 0 & 0 & 0 & 0 & 0\end{array}$

$\begin{array}{llllll}0 & 0 & 0 & 0 & 0 & 0\end{array}$

$\begin{array}{llllll}0 & 0 & 0 & 0 & 475 & 0\end{array}$

$\begin{array}{llllll}0 & 0 & 0 & 0 & 0 & 0\end{array}$

$\begin{array}{lllllll}0 & 0 & 0 & 0 & 1500 & -1500\end{array}$

$\begin{array}{lllllll}0 & 0 & 0 & 0 & 1500 & -1500\end{array}$

$\begin{array}{lllllll}0 & 0 & 0 & 0 & 1500 & -1500\end{array}$

$\begin{array}{lllllll}0 & 0 & 0 & 0 & 1500 & -1500\end{array}$

$\begin{array}{llllll}-1500 & -1500 & -1500 & -1500 & 0 & 475\end{array}$

$\begin{array}{llllll}1500 & 1500 & 1500 & 1500 & 3000 & 0\end{array}$

$\begin{array}{llllll}0 & 1500 & 1500 & 1500 & 1500 & 3000\end{array}$

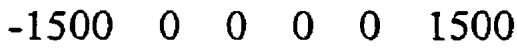

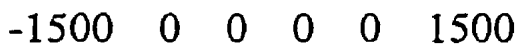

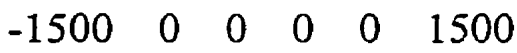

$\begin{array}{llllll}-1500 & 0 & 0 & 0 & 0 & 1500\end{array}$

$\begin{array}{llllll}475 & -1500 & -1500 & -1500 & -1500 & 0\end{array}$

$\begin{array}{llllll}0 & -1500 & 0 & 0 & 0 & 1500\end{array}$

$\begin{array}{llllll}1500 & 0 & 1500 & 1500 & 1500 & 3000\end{array}$

$\begin{array}{llllll}0 & -1500 & 0 & 0 & 0 & 1500\end{array}$

$\begin{array}{llllll}0 & -1500 & 0 & 0 & 0 & 1500\end{array}$

$\begin{array}{llllll}0 & -1500 & 0 & 0 & 0 & 1500\end{array}$

$\begin{array}{llllll}-1500 & 475 & -1500 & -1500 & -1500 & 0\end{array}$

$\begin{array}{llllll}0 & 0 & -1500 & 0 & 0 & 1500\end{array}$

$\begin{array}{lllllll}0 & 0 & -1500 & 0 & 0 & 1500\end{array}$

$\begin{array}{llllll}1500 & 1500 & 0 & 1500 & 1500 & 3000\end{array}$

$\begin{array}{llllll}0 & 0 & -1500 & 0 & 0 & 1500\end{array}$

$\begin{array}{llllll}0 & 0 & -1500 & 0 & 0 & 1500\end{array}$

$\begin{array}{llllll}-1500 & -1500 & 475 & -1500 & -1500 & 0\end{array}$ 


$$
\begin{array}{llllllll}
0 & 0 & 0 & -1500 & 0 & 1500 & & \\
0 & 0 & 0 & -1500 & 0 & 1500 & & \\
0 & 0 & 0 & -1500 & 0 & 1500 & & \\
1500 & 1500 & 1500 & 0 & 1500 & 3000 \\
0 & 0 & 0 & -1500 & 0 & 1500 & & \\
-1500 & -1500 & -1500 & 475 & -1500 & 0
\end{array}
$$

$\begin{array}{llllllll}0 & 0 & 0 & 0 & -1500 & 1500 & & \\ 0 & 0 & 0 & 0 & -1500 & 1500 & & \\ 0 & 0 & 0 & 0 & -1500 & 1500 & & \\ 0 & 0 & 0 & 0 & -1500 & 1500 & & \\ 1500 & 1500 & 1500 & 1500 & 0 & 3000 \\ -1500 & -1500 & -1500 & -1500 & 475 & 0\end{array}$

$$
\begin{array}{llllll}
0 & 0 & 0 & 0 & 0 & 0 \\
0 & 0 & 0 & 0 & 0 & 0 \\
0 & 0 & 0 & 0 & 0 & 0 \\
0 & 0 & 0 & 0 & 0 & 0 \\
0 & 0 & 0 & 0 & 0 & 0 \\
0 & 0 & 0 & 0 & 0 & 475
\end{array}
$$

// initial activation chosen by the system

initial activations

the activations:

$-136.500015-139.100021-161.040009-167.560013-235.200012-167.400009$ $-225.280014-185.600006-193.699982-172.500000-215.740005-367.429962$ $-487.549805-480.199921-426.240112-241.900009-218.279999-488.040009$ $-519.719910-397.600006-265.739990-380.799927-412.490021-307.800018$ $-361.759979-558.789978-317.519989-341.460083-382.500092-256.409943$ $-324.359985-328.600006-249.900055-250.740021-434.999908-435.600006$

// initial outputs

the outputs

0.1627370 .1585310 .1264760 .1180820 .0561260 .118282

0.0627750 .0973340 .0891220 .1120470 .0698580 .012019

0.0028700 .0031340 .0059710 .0520150 .0679030 .002853

0.0019530 .0083990 .0395860 .0102560 .0070340 .024278 


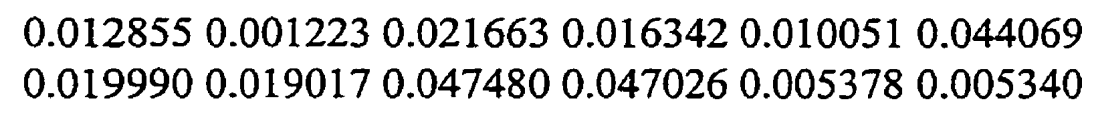

1000 iterations completed

// the final activation after 1000 iterations

the activations:

$30.356525-5.192744-47.893620-84.144035-140.938461-150.279877$

$-113.019150-287.490692-473.873627-649.023438-843.773682-1070.680664$

$-180.451660-356.239868-516.231567-636.669312-805.457397-1062.271606$

$-128.888748-211.460709-289.990265-442.986481-569.979248-655.941895$

$-63.722340-236.468277-270.334015-384.702301-503.552795-571.998352$

$-179.942276-417.925751-627.078979-860.128540-1148.140625-1380.642822$

// the final outputs

the outputs

0.1500760 .2844270 .0601480 .8670330 .1556100 .141443

0.2048540 .0307720 .0033800 .0004140 .0000400 .000003

0.1028990 .0137230 .0020360 .0004810 .0000630 .000003

0.1755680 .0732690 .0298900 .0048890 .0010690 .910381

0.3176340 .0553250 .0375430 .0097910 .0023700 .001044

0.9804650 .0065930 .0005390 .0000330 .0000010 .000000

the route :

I

4

6

cost of route is : 11.000000 